\title{
FINITE TIME EXTINCTION OF SUPERPROCESSES WITH CATALYSTS
}

\author{
By Donald A. Dawson ${ }^{1}$, Klaus Fleischmann ${ }^{2}$ and Carl MuelleR ${ }^{3}$ \\ Fields Institute, Weierstrass Institute for Applied Analysis and \\ Stochastics and University of Rochester
}

Consider a catalytic super-Brownian motion $X=X^{\Gamma}$ with finite variance branching. Here "catalytic" means that branching of the reactant $X$ is only possible in the presence of some catalyst. Our intrinsic example of a catalyst is a stable random measure $\Gamma$ on $\mathrm{R}$ of index $0<\gamma<1$. Consequently, here the catalyst is located in a countable dense subset of $\mathrm{R}$. Starting with a finite reactant mass $X_{0}$ supported by a compact set, $X$ is shown to die in finite time. We also deal with two other cases, with a power low catalyst and with a super-random walk on $Z^{d}$ with an i.i.d. catalyst.

Our probabilistic argument uses the idea of good and bad historical paths of reactant "particles" during time periods $\left[T_{n}, T_{n+1}\right)$. Good paths have a significant collision local time with the catalyst, and extinction can be shown by individual time change according to the collision local time and a comparison with Feller's branching diffusion. On the other hand, the remaining bad paths are shown to have a small expected mass at time $T_{n+1}$ which can be controlled by the hitting probability of point catalysts and the collision local time spent on them.

\section{Contents}

1. Introduction

1.1 Model 1: stable catalyst $\Gamma$ on $\mathrm{R}$

1.2 Model 2: i.i.d. uniform catalysts on the lattice $Z^{d}$

1.3 Model 3: a deterministic power law catalyst $\chi_{q}$

1.4 Finite time survival

1.5 Outline

2. Stable catalysts: main result

2.1 Preliminaries: some spaces

2.2 Branching rate functional $K$ and BCLT $L_{[W, \psi]}$

2.3 SBM $X$ with branching rate functional $K$

2.4 Main result: finite time extinction of $X^{\Gamma}$

Received September 1998; revised October 1999.

${ }^{1}$ Supported in part by a NSERC Grant and a Max Planck award.

${ }^{2}$ Supported in part by the DFG.

${ }^{3}$ Supported in part by an NSA grant.

AMS 1991 subject classifications. Primary 60J80; secondary 60J55, 60G57.

Key words and phrases. Catalytic super-Brownian motion, historical superprocess, critical branching, finite time extinction, finite time survival, measure-valued branching, random medium, good and bad paths, stopped measures, collision local time, comparison, stopped historical superprocess, branching rate functional, super-random walk, interacting Feller's branching diffusion, stable catalyst. 
3. An abstract finite time extinction criterion

3.1 Refinement: historical SBM $\widetilde{X}$

3.2 Dynkin's "stopped" measures $\tilde{X}_{\tau}$

3.3 The method of good and bad historical paths

3.4 Proof of the abstract criterion

3.5 Scaled comparison with Feller's branching diffusion

3.6 Proof of the comparison argument

4. Two applications of the abstract criterion

4.1 Power law catalyst $\chi_{q}$ (Model 3)

4.2 A point catalyst with dense locations

4.3 Nontrivial examples for finite time survival

5. Proof of the main result

5.1 Passing to a periodic catalyst

5.2 Completion of the proof of the main theorem

6 . The lattice model

1. Introduction. Recently a number of papers have dealt with branching in catalytic media. These are models of chemical or biological reactiondiffusion systems of two substances or species, respectively. One we call the catalyst, and the other the reactant. The latter we model by a super-Brownian motion (SBM) with "critical binary" branching, and its branching rate is given by the catalyst.

In this paper we verify finite time extinction of the reactant for three different types of catalysts, provided the reactant was started with a finite mass. We begin with explaining the most interesting of these catalysts.

1.1. Model 1: stable catalyst $\Gamma$ on R. Let $X^{\Gamma}=\left\{X_{t}^{\Gamma}: t \geq 0\right\}$ denote a continuous super-Brownian motion (SBM) with branching rate (catalyst) given by a stable random measure

$$
\Gamma=\sum_{i} \alpha_{i} \delta_{b_{i}}
$$

on the real line $\mathrm{R}$ with index $0<\gamma<1$ [for the latter, see (21) below].

At an intuitive level, this model can be explained as follows. A huge number of small reactant "particles" move independently according to Brownian motions in R. But if such a Brownian particle meets one of the point catalysts $\alpha_{i} \delta_{b_{i}}$ it may branch in a critical binary way. More precisely, branching is governed by the collision local time

$$
L_{[W, \Gamma]}(d s):=d s \sum_{i} \alpha_{i} \delta_{b_{i}}\left(W_{s}\right)
$$

in the spirit of [1] of the Brownian reactant particle with path $W$ and the stable random measure $\Gamma(d b)$ describing the catalyst. Note that

$$
L_{[W, \Gamma]}([0, t])=\sum_{i} \alpha_{i} L_{t}\left(b_{i}\right), \quad t \geq 0,
$$

where $L_{t}(b)$ denotes the Brownian local time at $b$ by time $t$. 
This process $X^{\Gamma}$ was introduced as a Markov process by Dawson and Fleischmann ([3], Lemma 2.3.5 and its application in Sections 2.4-2.5). The existence of a continuous version follows from [5], Theorem 1b. The clumping features of $X^{\Gamma}$ had been exhibited in [3] by a time-space-mass scaling limit theorem. In [8] the states $X_{t}^{\Gamma}$ of $X^{\Gamma}$ had been shown to be absolutely continuous measures. Finally, in [9], the so-called compact support property has been verified: if the finite initial measure $X_{0}^{\Gamma}$ has compact support, then the range of $X^{\Gamma}$ is compact, too.

Starting with a finite measure $X_{0}^{\Gamma}$, and given $\Gamma$, the total mass process $t \mapsto X_{t}^{\Gamma}(\mathrm{R})$ is a continuous martingale, hence has a.s. a limit as $t \uparrow \infty$ ([5], Proposition 3). The main purpose of the present paper is to show that if $X_{0}^{\Gamma}$ is of compact support, the process $X^{\Gamma}$ dies in finite time (Theorem 6 below), just as in the constant medium case (the formal $\gamma=1$ boundary case).

To illustrate the problems we encounter in the proof, we consider the following. Given the catalyst $\Gamma$ and starting $X^{\Gamma}$ with a unit mass concentrated at $a$, that is $X_{0}^{\Gamma}=\delta_{a}$, the probability of extinction of $X^{\Gamma}$ at time $t$ is given by

$$
P_{0, \delta_{a}}^{\Gamma}\left(X_{t}^{\Gamma}=0\right)=\exp \left[-v_{\infty}(0, a \mid t, \Gamma)\right],
$$

where for $\theta, t, \Gamma$ fixed, $v_{\theta}=v_{\theta}(\cdot, \cdot \mid t, \Gamma)=\left\{v_{\theta}(s, a \mid t, \Gamma):(s, a) \in[0, t] \times \mathrm{R}\right\}$ solves (formally) the following reaction-diffusion equation in the stable catalytic medium $\Gamma$ :

$$
-\frac{\partial}{\partial s} v_{\theta}=\frac{1}{2} \Delta v_{\theta}-\Gamma v_{\theta}^{2},\left.\quad v_{\theta}(s, a \mid t, \Gamma)\right|_{s=t} \equiv \theta \geq 0
$$

and $v_{\infty}:=\lim _{\theta \uparrow \infty} v_{\theta}$. Then, by Borel-Cantelli, it would suffice to show the following extinction property of solutions to (5):

$$
\lim _{t \uparrow \infty} \lim _{\theta \uparrow \infty} v_{\theta}(0, a \mid t, \Gamma)=0 .
$$

However, we do not know how to attack this problem analytically. Recall that the coefficient $\Gamma$ of the reaction term (reaction rate) in (5) is the generalized derivative of a (random) measure supported by a countable dense set in $R$, hence is highly singular.

Instead, to prove finite time extinction we will use some probabilistic arguments concerning the stochastic process $X^{\Gamma}$, inspired by Fleischmann and Mueller [20], which turns out to be a very flexible argument. (For another recent application, see [18].)

At the same time, via the log-Laplace connection of $X^{\Gamma}$ to the partial differential equation (5), our approach can be regarded as a probabilistic contribution to the study of asymptotic properties [such as (6)] of solutions to the reaction-diffusion equation (5) in the (random) heterogeneous singular medium $\Gamma$.

Equations as (5) have attracted some attention and are relevant in particular from an applied point of view; see, for example, [27], [28]. For reaction-diffusion equations in heterogenous media with different species and where reaction may be concentrated on bounded interfaces, see [24]. Note 
that reaction-diffusion equations arise in many branches of technology, for example, microelectronics.

The main ideas of our approach are as follows. First of all, since we start with an initial measure $X_{0}$ of compact support, and $X^{\Gamma}$ has the compact support property ([9]), we may "essentially" restrict our attention to a finite (space) interval $(-\mathrm{K}, \mathrm{K}] \subset \mathrm{R}$. Hence, by a stopping argument, the catalyst may be extended periodically outside $(-K, K]$. Next, the probability of extinction can be estimated below by using a smaller branching rate. Therefore, we remove all atoms $\alpha_{i} \delta_{b_{i}}$ of the catalyst with large "action weights" $\alpha_{i}$. Moreover, the action weights $\alpha_{i}$ belonging to $\left[2^{-n}, 2^{-n+1}\right.$ ) are replaced by $2^{-n}$, so that the corresponding atoms form Poisson point processes in $(-\mathrm{K}, \mathrm{K}]$ of intensity $c 2^{\gamma n}$ (with $c$ an appropriate constant). Finally, "large" distances between neighboring points of this Poisson point process are exceptional. Therefore, we may restrict to the situation where the empty intervals are at most of a size $\Delta_{n}$ (to be specified later). By the periodic expansion, then at the whole axis neighboring points are at most distance $\Delta_{n}$ apart. Altogether, we then want to verify finite time extinction of $X^{\Gamma}$ where the catalyst $\underline{\Gamma}$ is of the form $\sum_{n \geq 0} 2^{-n} \underline{\pi}_{n}$ where $\underline{\pi}_{n}$ is a periodic point measure with gaps between neighboring catalysts bounded by $\Delta_{n}$.

The central idea is to look for a sequence times $T_{1}<T_{2}<\cdots$ with finite accumulation point $T_{\infty}$ with the following property. At time $T_{n}$, we distinguish between "good and bad" historical paths of Brownian reactant particles, starting from the state $X_{T_{n}}^{\Gamma}$ at time $T_{n}$, as we now explain.

The "good" paths are those which have a "significant" collision local time with $2^{-n} \underline{\pi}_{n}$ on the time interval [ $T_{n}, T_{n+1}$ ) (so we take into account only that part $2^{-n} \underline{\pi}_{n}$ of $\left.\underline{\Gamma}\right)$. Consider the total mass of the good paths. For the continuous SBM with a uniform branching rate, the total mass process would have the same distribution as the (critical) Feller's branching diffusion which satisfies the one-dimensional stochastic equation

$$
d Z_{r}=\sqrt{2 Z_{r}} d W_{r}, \quad Z_{0} \geq 0
$$

(with $W$ a standard Brownian motion). It is well known that this diffusion is absorbed at 0 in finite time. In our catalytic case, the total mass of the good paths can in law be compared with Feller's branching diffusion. But now its time scale during $\left[T_{n}, T_{n+1}\right)$ is, roughly speaking, individually given by the collision local times of the good paths with the catalytic medium $2^{-n} \underline{\pi}_{n}$. Since these collision local times are "significant" on the good paths, it follows that the total mass of the good paths dies out by time $T_{n+1}$ with high probability.

The remaining "bad" paths may not die out by time $T_{n+1}$, but we can estimate the probability that this mass is larger than a certain size at time $T_{n+1}$, by using Markov's inequality and the simple but powerful expectation formula for (historical) superprocesses. Then we need to derive some estimates concerning hitting probabilities of a neighboring point catalyst from $2^{-n} \underline{\pi}_{n}$, and the Brownian (collision) local time spent on it. 
1.2. Model 2: i.i.d. uniform catalysts on the lattice $Z^{d}$. In the other two models we discuss, the basic ideas of distinguishing between good and bad historical paths, and how to handle them, are the same. So here we only introduce the models and indicate how to classify the paths.

For the second model, we replace the phase space $\mathrm{R}$ by the lattice $\mathrm{Z}^{d}$, and Brownian motion by a continuous time simple random walk in $Z^{d}$. The catalysts $\varrho=\left\{\varrho_{b}: b \in Z^{d}\right\}$ are i.i.d. random variables, uniform in the interval $(0,1)$. So here the catalysts are present everywhere but again their action weights $\varrho_{b}$ fluctuate randomly. Given the catalyst $\varrho$, the super-random walk $X^{\varrho}$ with catalyst $\varrho$ can be defined by the following system of interacting diffusions in $\mathrm{R}_{+}$:

$$
d X_{t}^{\varrho}(b)=\frac{1}{2} \Delta X_{t}^{\varrho}(b) d t+\sqrt{2 \varrho_{b} X_{t}^{\varrho}(b)} d W_{t}(b),
$$

$t>0, b \in Z^{d}$, where $\Delta$ is the discrete Laplacian in $Z^{d}$, and $\left\{W(b): b \in Z^{d}\right\}$ is a family of independent one-dimensional (standard) Brownian motions. In other words, we consider interacting Feller's branching diffusions $X^{\varrho}$ on $Z^{d}$ in the catalytic medium $\varrho$. Note that given the catalyst $\varrho$, for each bounded initial state $X_{0}^{\varrho}$, a unique strong solution $X^{\varrho}$ of (8) exists, that is, (8) is a well-posed problem [31].

By the discreteness of $Z^{d}$, and since masses can be arbitrarily small in superprocesses, one does not expect that the compact support property holds. Therefore, as opposed to Model 1, the super-random walk $X^{\varrho}$ with catalyst $\varrho$ may be influenced by large regions, where the catalysts are small. However, calling paths "bad" which reach such a region, these paths should have a small expected mass, and we will be able to show the finite time extinction property for $X^{\varrho}$ along the lines indicated.

Intuitively, it is clear that for these arguments the special form of the uniform density is not important. But boundedness away from zero and infinity of the density of catalytic mass at a site seems to be essential.

1.3. Model 3: a deterministic power law catalyst $\chi_{q}$. For the moment, consider the continuous SBM with phase space $\mathrm{R}$ and uniform branching rate, except on $(-1,1)$. More precisely, we consider the branching rate $\chi=\mathbf{1}_{R \backslash(-1,1)}$. As we will see in the next subsection, if $X_{0}((-1,1))>0$, then this superprocesses does not die in finite time.

Motivated by this, for a fixed constant $q>0$, we consider the truncated power law branching rate

$$
\chi(b)=\chi_{q}(b):=|b|^{q} \wedge 1, \quad b \in \mathrm{R}
$$

(see Figure 1). The SBM $X^{\chi}$ with power law catalyst $\chi_{q}$ is covered, for instance, by the class of models dealt with in [26].

We show that starting with a finite initial mass, $X^{\chi}$ dies in finite time, just as in the constant branching rate case, despite the "depression" of branching rate close to the origin, even if $q$ is very large. Here the good historical paths 

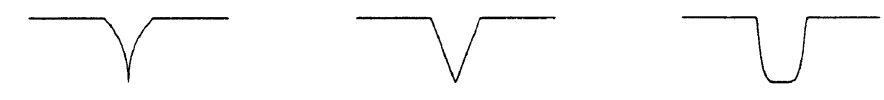

$$
\chi_{1 / 3}(b)=|b|^{1 / 3} \wedge 1 \quad \chi_{1}(b)=|b| \wedge 1 \quad \chi_{5}(b)=|b|^{5} \wedge 1
$$

FIG. 1. Variants of the power law catalyst.

are those which do not spend too much time near 0 , where the catalytic mass is small.

1.4. Finite time survival. If we change Model 1 so that the catalysts are not dense, then the reactant mass fails to die out in finite time. In fact, if $I \neq \varnothing$ is an open interval without catalysts, then a corresponding catalytic SBM $X$ is bounded below by the heat flow in $I$ with absorption at the boundary $\partial I$, starting with $X_{0}(\cdot \cap I)$. If now $X_{0}(I)>0$, then the $L^{1}$-norm of that heat solution decays according to $\left\langle X_{0}, \varphi_{\lambda}\right\rangle e^{-\lambda t}$ with $\lambda>0$ the first eigenvalue of $\frac{1}{2} \Delta$ on $I$, and $\varphi_{\lambda}$ the corresponding eigenfunction, hence is (strictly) positive at any time $t$, that is $X_{t}(I)>0$ for all $t$.

Note that catalytic SBMs with a gap cover the single point catalytic SBM, where survival for all finite times was known from Fleischmann and Le Gall [19].

It would be interesting to establish conditions on the catalytic medium which are necessary and sufficient for extinction in finite time. Unfortunately, our methods seem to be too crude for this. But in Section 4.3 below we will complement our finite time extinction results by two nontrivial examples of catalytic SBMs in $\mathrm{R}$ for which finite time extinction is violated. In fact, in the first case, we replace the power law catalyst by one which has a still "deeper depression" but the catalytic density is nevertheless positive everywhere except at 0 . In the second example, the catalyst is dense at R but "very sparse" in the sense that it can be considered as a $\gamma=0$ boundary case of a stable catalyst with index $\gamma$.

REMARK 1 (Decomposition of initial measures). Suppose a finite decomposition $\mu=\sum_{i} \mu_{i}$ of the initial measure is given. If we can show finite time extinction for each initial measure $X_{0}=\mu_{i}$ then the branching property implies finite time extinction for $X_{0}=\mu$.

1.5. Outline. To give a precise meaning to the above ideas, some technical problems have to be overcome. For instance, to have access to reactant particle paths, we will work with the historical catalytic SBM $\tilde{X}^{\Gamma}$ instead of $X^{\Gamma}$. Or, since we want to use time scales of individual reactant particles, we will exploit Dynkin's [14] framework of "stopped" historical superprocesses.

The outline of the paper is as follows. In the next section we recall the model of continuous $S B M X$ in $\mathrm{R}^{d}$ with branching rate functional $K$ as provided in [5] (this goes back to [14]). Then $K$ is specialized to be the Brownian collision local time $K=L_{[W, \psi]}$ of a (deterministic) locally finite measure $\psi$ (catalyst) on R, 
also taken from [5]. Further specialized to $\psi=\Gamma$, our main result, Theorem 6 below, can be formulated.

In Section 3 we first recall the historical SBM $\tilde{X}$ in $\mathrm{R}^{d}$ with branching rate functional $K$. For this model, we give an abstract sufficiency criterion (Theorem 10) for finite time extinction based on the idea of good and bad paths. For the extinction of good paths, a comparison with Feller's branching diffusion is provided (Proposition 12), as a refinement of an argument in [20]. This makes use of Dynkin's concept of (individually) "stopped” historical superprocesses.

Section 4 is devoted to two one-dimensional applications of the abstract criterion:

(a) the power law catalyst $\chi_{q}$ of Model 3, and

(b) a (deterministic) point catalyst $\underline{\Gamma}=\sum_{n \geq N} 2^{-n} \underline{\pi}_{n}$ with dense locations and gaps between neighboring catalysts in $\underline{\pi}_{n}$ bounded by some $\Delta_{n}$.

In Section 5 we prove our main theorem, the finite time extinction for the SBM $X^{\Gamma}$ with a stable catalytic rate $\Gamma$ (Model 1$)$. In fact, by periodic expansion and a comparison argument, we reduce the problem to the case (b) above.

Finally, in Section 6, finite time extinction for the super-random walk $X^{\varrho}$ with i.i.d. uniform catalysts is derived.

For a brief introduction to the results presented in this paper, see [21]. For recent surveys on catalytic branching models, we recommend [6] and [25].

We restrict our attention completely to finite measure-valued reactant processes, although otherwise one could ask for local extinction in finite time (for a study of this notion, see also [16] and references therein).

2. Stable catalysts: main result. Here we carefully introduce the continuous SBM $X$ in $\mathrm{R}^{d}$ with a sufficiently nice branching rate functional $K$. After specializations to Model 1, we will formulate our main result, Theorem 6 below.

2.1. Preliminaries: some spaces. Measurability is always meant with respect to the related Borel fields. The lower index + refers to the subset of all nonnegative members of a set.

Let $\mathscr{B}\left[E_{1}, E_{2}\right]$ denote the set of all measurable mappings $f: E_{1} \rightarrow E_{2}$ where $E_{1}, E_{2}$ are topological spaces. Write $\mathscr{B}\left[E_{1}\right]$ instead of $\mathscr{B}\left[E_{1}, E_{2}\right]$ if $E_{2}=\mathrm{R}$, the real line, and only $\mathscr{B}$ if additionally $E_{1}=\mathrm{R}^{d}, d \geq 1$.

If we restrict our consideration to continuous functions $f$, the letter $\mathscr{B}$ is replaced by $\mathscr{b}$ in the respective cases. If we restrict to bounded functions, we write $b \mathscr{B}$ and $b \mathscr{C}$, etc.

Fix a dimension $d \geq 1$, and a constant $p>d$, and introduce the reference function

$$
\phi_{p}(b):=\left(1+|b|^{2}\right)^{-p / 2}, \quad b \in \mathrm{R}^{d},
$$

of $p$-potential decay at infinity. Denote by $\mathscr{B}^{p}$ the set of all those $\varphi \in \mathscr{B}$ such that $|\varphi| \leq c_{\varphi} \phi_{p}$ for some constant $c_{\varphi}$. 
Write $\langle\mu, f\rangle$ for the integral $\int \mu(d b) f(b)$. Let $\mathscr{M}_{p}=\mathscr{M}_{p}\left[\mathrm{R}^{d}\right]$ denote the set of all (nonnegative) measures $\mu$ defined on $\mathrm{R}^{d}$ satisfying $\left\langle\mu, \phi_{p}\right\rangle<\infty$. We endow this set $\mathscr{M}_{p}$ of $p$-tempered measures with the weakest topology such that all the maps $\mu \mapsto\langle\mu, \varphi\rangle$ are continuous, where $\varphi \geq 0$ is continuous and of compact support, or $\varphi=\phi_{p}$. The set of all finite measures on a Polish space $E$ is denoted by $\mathscr{M}_{f}[E]$ and equipped with the topology of weak convergence. Write $\|\mu\|$ for the total mass $\mu(E)=\langle\mu, 1\rangle$ of $\mu \in \mathscr{M}_{f}[E]$.

Set $\mathscr{M}_{f}=\mathscr{M}_{f}\left[\mathrm{R}^{d}\right]$, and denote by $\mathscr{M}\left[\mathrm{R}_{+} \times E\right]$ the set of all measures $\eta$ defined on $\mathrm{R}_{+} \times E$ such that $\eta([0, t] \times E)<\infty$ for all $t>0$.

With $c$ we always denote a positive constant which may be different at various places. On the other hand, constants $c_{i}$ are fixed within each subsection.

2.2. Branching rate functional $K$ and $B C L T L_{[W, \psi]}$. Let $W=\left[W, \Pi_{s, a}\right.$, $s \geq 0, a \in \mathrm{R}^{d}$ ] denote the (standard) Brownian motion in $\mathrm{R}^{d}$, on canonical path space $\mathscr{C}\left[\mathrm{R}_{+}, \mathrm{R}^{d}\right]$ of continuous functions $w$.

REMARK 2 (Inhomogeneous setting). Although Brownian motion is timehomogeneous, we use this inhomogeneous setting, and we read $\Pi_{s, a} \varphi\left(W_{t}\right)$ as 0 if $s>t$. This formalism looks artificial since charging the paths before time $s$ does not change the laws $\Pi_{s, a}$. The advantage becomes clear when we work with historical SBM. Note that the measure $\Pi_{s, a}$ is concentrated on the set of paths $\left\{w \in \mathscr{C}\left[\mathbf{R}_{+}, \mathbf{R}^{d}\right]: w_{s}=a\right\}$.

Write $p$ for the continuous transition density function of $W$,

$$
p_{t}(a, b)=p_{t}(b-a)=(2 \pi t)^{-d / 2} \exp \left[-\frac{(b-a)^{2}}{2 t}\right], \quad t>0, a, b \in \mathrm{R}^{d}
$$

and

$$
\Pi_{\eta}:=\int \eta(d s, d a) \Pi_{s, a}, \quad \eta \in \mathscr{M}\left[R_{+} \times R^{d}\right]
$$

for the "law" of $W$ starting at time $s$ in a point $a$ where $(s, a)$ is "distributed" according to the measure $\eta$.

For convenience, we introduce the following definition.

DEFINITION 3 (Branching rate functional). A continuous additive functional $K=K_{[W]}$ of Brownian motion $W$ is called a branching rate functional in $\mathbf{K}^{\nu}$, for some $\nu>0$, if the following two conditions hold:

(a) It is locally admissible, that is,

$$
\sup _{a \in R^{d}} \Pi_{s, a} \int_{s}^{t} K(d r) \phi_{p}\left(W_{r}\right) \underset{\substack{s \rightarrow r_{0} \\ t \rightarrow r_{0}}}{\longrightarrow} 0, \quad r_{0} \geq 0 .
$$

(For nonadmissible functionals, we refer to [7].) 
(b) For each $N$ there is a constant $c_{N}>0$ such that

$$
\Pi_{s, a} \int_{s}^{t} K(d r) \phi_{p}^{2}\left(W_{r}\right) \leq c_{N}|t-s|^{\nu} \phi_{p}(a), \quad 0 \leq s \leq t \leq N, a \in \mathrm{R}^{d} .
$$

To come to our main example of a branching rate functional, consider for the moment $d=1$ and fix $\psi \in \mathscr{M}_{p}$. Intuitively,

$$
L_{[W, \psi]}(d r):=d r \int \psi(d b) \delta_{b}\left(W_{r}\right)
$$

is the Brownian collision local time (BCLT) of $\psi$. From [5], Corollary 2, page 257, we immediately get the following statement. (For the more general case if $\varrho$ is also time-dependent or, in particular a path of ordinary SBM, we refer to [17] and references therein.)

Lemma 4 (Brownian collision local time of $\varrho$ ). Fix $d=1$ and $\psi \in \mathscr{M}_{p}$. The Brownian collision local time $L_{[W, \psi]}$ of $\psi$ makes sense nontrivially as a continuous additive functional of (one-dimensional) Brownian motion $W$, and it is a branching rate functional in $\mathbf{K}^{\nu}$ with $\nu=1 / 2$.

2.3. SBM $X$ with branching rate functional $K$. A slight modification of Proposition 12 (page 230) and Theorem 1 (page 234) in [5] gives the following lemma.

LEMMA 5 (Continuous SBM with branching rate functional $K$ ). Fix a dimension $d \geq 1$, and $K \in \mathbf{K}^{\nu}$ for some $\nu>0$.

(a) (Existence) There exists a continuous $\mathscr{M}_{f}$-valued (time-inhomogeneous) Markov process $X=\left[X, P_{s, \mu}, s \geq 0, \mu \in \mathscr{M}_{f}\right]$ with Laplace functional

$$
P_{s, \mu} \exp \left\langle X_{t},-\varphi\right\rangle=\exp \langle\mu,-v(s, \cdot \mid t)\rangle \text {, }
$$

$0 \leq s \leq t, \mu \in \mathscr{M}_{f}, \varphi \in b \mathscr{B}_{+}$, where for $t, \varphi$ fixed, $v=v(\cdot, \cdot \mid t) \geq 0$ is uniquely determined by the log-Laplace equation

$$
v(s, a)=\Pi_{s, a}\left[\varphi\left(W_{t}\right)-\int_{s}^{t} K(d r) v^{2}\left(r, W_{r}\right)\right]
$$

$0 \leq s \leq t, a \in \mathrm{R}^{d}$.

(b) (Modification) To each $\eta \in \mathscr{M}\left[\mathrm{R}_{+} \times \mathrm{R}^{d}\right]$, there is an $\mathscr{M}_{f}$-valued Markov process $\left[X, P_{\eta}\right]$ such that

$$
P_{\eta} \exp \left\langle X_{t},-\varphi\right\rangle=\exp \langle\eta,-v(\cdot, \cdot \mid t)\rangle, \quad t \geq 0,
$$

with $v(s, \cdot \mid t)$ from (a) if $0 \leq s \leq t$, and $v(s, \cdot \mid t)=0$ otherwise. 
(c) (Moments) $\left[X, P_{s, \mu}\right]$ has finite moments of all orders. In particular, for $\eta \in \mathscr{M}\left[\mathrm{R}_{+} \times \mathrm{R}^{d}\right]$ and $\varphi_{1}, \varphi_{2} \in b \mathscr{B}_{+}$, as well as $t_{1}, t_{2} \geq 0$,

$$
\begin{gathered}
P_{\eta}\left\langle X_{t_{1}},-\varphi_{1}\right\rangle=\Pi_{\eta} \varphi_{1}\left(W_{t_{1}}\right), \\
\operatorname{Cov}_{\eta}\left[\left\langle X_{t_{1}}, \varphi_{1}\right\rangle,\left\langle X_{t_{2}}, \varphi_{2}\right\rangle\right] \\
=2 \Pi_{\eta} \int K(d r) \mathbf{1}_{\left\{r \leq t_{1} \wedge t_{2}\right\}}\left[\Pi_{r, W_{r}} \varphi_{1}\left(W_{t_{1}}\right)\right]\left[\Pi_{r, W_{r}} \varphi_{2}\left(W_{t_{2}}\right)\right] .
\end{gathered}
$$

(Recall that for formulas as in (c) the convention is in force that $\Pi_{s, a} \varphi\left(W_{t}\right)=0$ if $s>t$, introduced in Remark 2.)

This superprocess $X$ is said to be the continuous $S B M$ with branching rate functional $K$. Note that the lemma in particular applies in the case of a BCLT $K=L_{[W, \psi]}$ according to Lemma 4, resulting in a time-homogeneous Markov process.

2.4. Main result: finite time extinction of $X^{\Gamma}$. Let $d=1$. Fix a constant $0<\gamma<1$, and a (not necessarily normalized) Lebesgue measure $\ell$ on $\mathrm{R}$. The stable catalyst $(\Gamma, \mathbb{P})$ is by definition the stable random measure on $\mathrm{R}$ with Laplace functional

$$
\mathbb{P} \exp \langle\Gamma,-\varphi\rangle=\exp \left[-\int \ell(d b) \varphi^{\gamma}(b)\right], \quad \varphi \in \mathscr{B}_{+} .
$$

Recall that $\Gamma$ has independent increments, and that it allows a representation

$$
\Gamma=\sum_{i} \alpha_{i} \delta_{b_{i}}
$$

with weights $\alpha_{i}>0$, and where the set of locations $b_{i}$ is dense in R, with P-probability 1.

We now additionally require $p>1 / \gamma$ [for the exponent $p$ of potential decay occurring in the reference function (10)]. Then by (21), the realizations of the catalyst $\Gamma$ belong $\mathbb{P}$-almost surely to $\mathscr{M}_{p}$. Hence we may apply the constructions of the previous two subsections to introduce the continuous $S B M$ $X^{\Gamma}=\left[X^{\Gamma}, P_{s, \mu}^{\Gamma}, s \geq 0, \mu \in \mathscr{M}_{f}\right]$ with stable catalyst $\Gamma$. More precisely, we use the so-called quenched approach: first a realization $\Gamma$ of the catalytic medium is selected according to $\mathbb{P}$, and then, given $\Gamma$, the continuous time-homogeneous Markov process $X^{\Gamma}$ evolves, governed by the BCLT $L_{[w, \Gamma]}$.

Note that by a formal differentiation of the log-Laplace equation (17) with $K=L_{[w, \Gamma]}$ of (15), using the semigroup of $W$, and replacing $\varphi$ by the constant function $\theta$, we get back the reaction-diffusion equation (5) in the catalytic medium $\Gamma$.

Now we are in a position to formulate our main result. Recall that $d=1$.

THEOREM 6 (Finite time extinction of $X^{\Gamma}$ ). Fix $\mu \in \mathscr{M}_{f}$ with compact support. For $\mathbb{P}$-almost all $\Gamma$ the following holds:

$$
P_{0, \mu}^{\Gamma}\left(X_{t}^{\Gamma}=0 \text { for some } t\right)=1 .
$$


The proof of this theorem needs some preparation and is postponed until Section 5 .

It is reasonable to expect that in some " $\gamma=0$ boundary cases" finite time extinction is violated. More precisely, think of a catalyst which is a random measure with independent increments which Lévy measure $\nu$ on $R_{+}$is infinite but has a density with a growth to infinity at 0 of order $r^{-1} L(r)$ as $r \downarrow 0$ with $L$ an appropriate slowly varying function (recall that in the $\gamma$-stable case the density's increase is of order $r^{-1-\gamma}$ as $r \downarrow 0$ ). See Corollary 17(ii) below.

3. An abstract finite time extinction criterion. The purpose of this section is to establish a general sufficient criterion for extinction in finite time for a SBM $X$ in $\mathrm{R}^{d}$ with branching rate functional $K$ (see Theorem 10 below). The central idea is to divide a finite time interval into an infinite number of stages in such a way that all of the mass will be dead at the end of all these stages. For this purpose, at each stage we distinguish between good and bad historical paths. The good paths accumulate a "significant" rate of branching, so that they die by the next stage, with high probability. The remaining bad paths may not die, but by assumption they carry a small expected mass at the beginning of the next stage.

3.1. Refinement: historical $S B M \widetilde{X}$. To realize this concept, we have to pass to a "historical" setting. That is, the measures $X_{t}(d b)$ on $\mathrm{R}^{d}$ are thought to be projections of measures $\widetilde{X}_{t}(d w)$ where $w$ is a continuous function on the interval $[0, t]$. Heuristically, a particle in $X_{t}$ with position $b$ is additionally equipped with a path $w:[0, t] \rightarrow \mathrm{R}^{d}$ with terminal point $w_{t}=b$, which gives the spatial past history of the particle and its ancestors. (For a more detailed exposition, we refer, e.g., to [20].)

Equip $\mathbf{C}:=\mathscr{C}\left[\mathrm{R}_{+}, \mathrm{R}^{d}\right]$ with the topology of uniform convergence on all compact subsets of $\mathrm{R}_{+}$. To each $w \in \mathbf{C}$ and $t \geq 0$, we associate the stopped path $w^{t} \in \mathbf{C}$ by setting $w_{s}^{t}:=w_{t \wedge s}, s \geq 0$. Write $\overline{\mathbf{C}}^{t}$ for the closed subspace of $\mathbf{C}$ of all these paths stopped at time $t$. Note that $\mathbf{C}^{t}$ could be regarded as $\mathscr{C}\left[[0, t], \mathrm{R}^{d}\right]$ (as we did in the previous paragraph) and $\mathbf{C}^{0}$ as $\mathrm{R}^{d}$.

To every $w \in \mathbf{C}$ we associate the corresponding stopped path trajectory $\tilde{w}$ by setting $\widetilde{w}_{t}:=w^{t}, t \geq 0$. Writing $\|\cdot\|_{\infty}$ for the supremum norm, for $0 \leq s \leq t$ we get

$$
\left\|\widetilde{w}_{t}-\widetilde{w}_{s}\right\|_{\infty}=\left\|w^{t}-w^{s}\right\|_{\infty}=\sup _{s \leq r \leq t}\left|w_{r}-w_{s}\right| \rightarrow 0 \quad \text { as } t-s \downarrow 0 .
$$

Hence, $\tilde{w}$ belongs to $\mathscr{C}\left[\mathbf{R}_{+}, \mathbf{C}\right]$.

The image of the Brownian motion $W$ under the map $w \mapsto \widetilde{w}$ is called the Brownian path process

$$
\widetilde{W}=\left[\widetilde{W}, \widetilde{\Pi}_{s, w}, s \geq 0, w \in \mathbf{C}^{s}\right] .
$$

That is, at time $s$ we start with a path $w=\widetilde{W}_{s}$ stopped at time $s$, and let a path trajectory $\left\{\widetilde{W}_{t}: t \geq s\right\}$ evolve with law $\widetilde{\Pi}_{s, w}$ determine by the path $\left\{W_{t}: t \geq s\right\}$ starting at time $s$ from $w_{s}$. 
Note that if $K$ belongs to $\mathbf{K}^{\nu}$ for some $\nu>0$, then $K$ is also a continuous additive functional with respect to the Brownian path process $\widetilde{W}$.

Set

$$
\mathrm{R}_{-} \widehat{\times} \mathbf{C}^{\bullet}:=\left\{(s, w): s \in \mathbf{R}_{+}, w \in \mathbf{C}^{s}\right\}
$$

and write $\mathscr{M}\left[\mathrm{R}_{+} \widehat{\times} \mathbf{C}^{\bullet}\right]$ for the set of all measures $\eta$ on $\mathbf{R}_{+} \widehat{\times} \mathbf{C} \cdot$ which are finite if restricted to a finite time interval. Moreover, let

$$
\widetilde{\Pi}_{\eta}:=\int \eta(d s, d w) \widetilde{\Pi}_{s, w}, \quad s \geq 0, \eta \in \mathscr{M}\left[\mathrm{R}_{+} \widehat{\times} \mathbf{C}^{\cdot}\right] .
$$

$W$ can be constructed from $\widetilde{W}$ by projection: $W_{t}:=\left(\widetilde{W}_{t}\right)_{t}$. this will often be used in the sequel.

Now we give the following historical version of Lemma 5, which follows from a modification of Propositions 1 (page 225), 12 (page 230) and Lemma 4 (page 232) in [5].

Proposition 7 (Historical SBM with branching rate functional $K$ ). Let $d \geq$ 1 , and fix $K \in \mathbf{K}^{\nu}$ for some $\nu>0$.

(a) (Existence) There exists a (time-inhomogeneous) Markov process

$$
\tilde{X}=\left[\tilde{X}, \widetilde{P}_{s, \mu}, s \geq 0, \mu \in \mathscr{M}_{f}\left[\mathbf{C}^{s}\right]\right]
$$

with states $\tilde{X}_{t} \in \mathscr{M}_{f}\left[\mathbf{C}^{t}\right], t \geq s$, and with Laplace functional

$$
\widetilde{P}_{s, \mu} \exp \left\langle\tilde{X}_{t},-\varphi\right\rangle=\exp \langle\mu,-v(s, \cdot \mid t)\rangle,
$$

$0 \leq s \leq t, \mu \in \mathscr{M}_{f}\left[\mathbf{C}^{s}\right], \varphi \in b \mathscr{B}_{+}[\mathbf{C}]$, where for $t, \varphi$ fixed, $v=v(\cdot, \cdot \mid t) \geq 0$ is uniquely determined by the log-Laplace equation

$$
v\left(s, \omega_{s}\right)=\widetilde{\Pi}_{s, \omega_{s}}\left[\varphi\left(\tilde{W}_{t}\right)-\int_{s}^{t} K(d r) v^{2}\left(r, \tilde{W}_{r}\right)\right],
$$

$0 \leq s \leq t, \omega_{s} \in \mathbf{C}^{s}$

(b) (Modification) To each $\eta \in \mathscr{M}\left[\mathrm{R}_{+} \widehat{\times} \mathbf{C}^{\bullet}\right]$ there is a Markov process $\left[\widetilde{X}, \widetilde{P}_{\eta}\right]$ with states $\tilde{X}_{t} \in \mathscr{M}_{f}\left[\mathbf{C}^{t}\right]$ and such that

$$
\widetilde{P}_{\eta} \exp \left\langle\tilde{X}_{t},-\varphi\right\rangle=\exp \langle\eta,-v(\cdot, \cdot \mid t)\rangle, \quad t \geq 0,
$$

with $v(s, \cdot \mid t)$ from (a) if $0 \leq s \leq t$, and $v(s, \cdot \mid t)=0$ otherwise.

(c) (Moments) $\left(\widetilde{X}, \widetilde{P}_{s, \mu}\right)$ has finite moments of all orders. In particular, for $\eta \in \mathscr{M}\left[\mathrm{R}_{+} \widehat{\times} \mathbf{C}^{\cdot}\right]$ and $\varphi_{1}, \varphi_{2} \in b \mathscr{B}_{+}[\mathbf{C}]$, as well as $t_{1}, t_{2} \geq 0$,

$$
\begin{gathered}
\widetilde{P}_{\eta}\left\langle\widetilde{X}_{t_{1}}, \varphi_{1}\right\rangle=\widetilde{\Pi}_{\eta} \varphi_{1}\left(\widetilde{W}_{t_{1}}\right), \\
\widetilde{\operatorname{Cov}}_{\eta}\left[\left\langle\widetilde{X}_{t_{1}}, \varphi_{1}\right\rangle,\left\langle\widetilde{X}_{t_{2}}, \varphi_{2}\right\rangle\right] \\
=2 \widetilde{\Pi}_{\eta} \int K(d r) \mathbf{1}_{\left\{r \leq t_{1} \wedge t_{2}\right\}}\left[\widetilde{\Pi}_{r, \widetilde{W}_{r}} \varphi_{1}\left(\widetilde{W}_{t_{1}}\right)\right]\left[\widetilde{\Pi}_{r, \widetilde{W}_{r}} \varphi_{2}\left(\widetilde{W}_{t_{2}}\right)\right] .
\end{gathered}
$$


(Recall once more that for formulas as in (c) we use the convention introduced in Remark 2 which by projection also applies for historical Brownian motion.)

We call this superprocess $\tilde{X}$ the historical $S B M$ with branching rate function $K$.

Of course, $X$ can be gained back from $\tilde{X}$ by projection:

$$
X_{t}=\widetilde{X}_{t}\left(\left\{w \in \mathbf{C}^{t}: w_{t} \in(\cdot)\right\}\right) .
$$

3.2. Dynkin's “stopped" measures $\tilde{X}_{\tau}$. We also have to recall Dynkin's $[14,15]$ concept of "stopped" historical superprocesses. We have two reasons for this. First, to handle also the lattice Model 2, we have to allow the times $T_{1}<T_{2}<\cdots$ mentioned in Section 1.1 to be Brownian stopping times. The second reason is that we intend to scale the SBM along individual particles' trajectories according to their accumulated rate of branching. Note that this is related to the work of Perkins [29] where stochastic integrals are developed along individual particles' trajectories in the historical superprocess.

Roughly speaking, if $\tau$ is a Brownian stopping time, Dynkin's "stopped" measure $\widetilde{X}_{\tau}$ describes the population one gets, if each (individual) reactant path is stopped in the moment $\tau$. More precisely, $\tilde{X}_{\tau}$ is a random measure in $\mathscr{M}\left[\mathrm{R}_{-} \widehat{\times} \mathbf{C}^{\cdot}\right]$. For a detailed development, we refer to [14], Section 1.5 and [15], Section 1.10. For convenience, here we collect only the following facts.

Let $\tau_{t}, t \geq 0$, be stopping times with respect to the (natural) filtration of Brownian motion $W$, satisfying $\tau_{s} \leq \tau_{t}$ if $s \leq t$. Then there is a family

$$
\left\{\tilde{X}_{\tau_{t}}: t \geq 0\right\},
$$

of random measures in $\mathscr{M}\left[\mathrm{R}_{+} \widehat{\times} \mathbf{C}^{\cdot}\right]$, the so-called "stopped" historical SBM related to the family of Brownian stopping times $\left\{\tau_{t}: t \geq 0\right\}$. This family satisfies the so-called special Markov property, which roughly says the following: for $s \geq 0$, let $\mathscr{G}_{\tau_{s}}$ denote the pre- $\tau_{s} \sigma$-field (concerning the historical superprocess $\widetilde{X}$ ). Given $\mathscr{G}_{\tau_{s}}$, hence in particular $\widetilde{X}_{\tau_{s}}=: \vartheta$, the "stopped" process $\left\{\widetilde{X}_{\tau_{t}}: t \geq s\right\}$ "starts anew" ([14], Theorem 1.6 and [15], Theorem 1.5), namely based on the law $\widetilde{P}_{\vartheta}$.

Similarly, the notation of a sequence $\left\{\tilde{X}_{\tau_{n}}: n \geq 1\right\}$ of "stopped" measures related to Brownian stopping times $\tau_{1} \leq \tau_{2} \leq \cdots$ can be introduced.

In formal analogy with Proposition $7(\mathrm{~b})$, we get the following log-Laplace representation for the stopped historical SBM (see [15], Theorem 1.4). For $t \geq 0$ and $\eta \in \mathscr{M}\left[\mathrm{R}_{+} \widehat{\times} \mathbf{C} \cdot\right]$ as well as $\varphi$ in $b \mathscr{B}_{+}\left[\mathrm{R}_{+} \times \mathbf{C}\right]$,

$$
\widetilde{P}_{\eta} \exp \left\langle\tilde{X}_{\tau_{t}},-\varphi\right\rangle=\exp \left\langle\eta,-v_{t}\right\rangle
$$

where $v_{t}$ solves

$$
v_{t}\left(s, \omega_{s}\right)=\widetilde{\Pi}_{s, \omega_{s}} \mathbf{1}_{\left\{s \leq \tau_{t}\right\}}\left[\varphi\left(\tau_{t}, \widetilde{W}_{\tau_{t}}\right)-\int_{s}^{\tau_{t}} K(d r) v_{t}^{2}\left(r, \widetilde{W}_{r}\right)\right] .
$$


In particular, the following first two moment formulas hold ([14], (1.50a)):

$$
\begin{aligned}
\widetilde{P}_{\eta}\left\langle\widetilde{X}_{\tau_{t}}, \varphi\right\rangle & =\widetilde{\Pi}_{\eta} \varphi\left(\tau_{t}, \widetilde{W}_{\tau_{t}}\right), \\
\tilde{\operatorname{Var}}_{\eta}\left\langle\widetilde{X}_{\tau_{t}}, \varphi\right\rangle & =2 \widetilde{\Pi}_{\eta} \int K(d r) \mathbf{1}_{\left\{r \leq \tau_{t}\right\}}\left[\widetilde{\Pi}_{r, \widetilde{W}_{r}} \varphi\left(\tau_{t}, \widetilde{W}_{\tau_{t}}\right)\right]^{2} .
\end{aligned}
$$

Finally, we adopt the convention to write, for (measurable) subsets $E$ of $\mathbf{C}$,

$$
\tilde{X}_{\tau_{t}}(E):=\tilde{X}_{\tau_{t}}\left(\left(\mathrm{R}_{+} \widehat{x} \mathbf{C}^{\bullet}\right) \cap\left(\mathrm{R}_{+} \times E\right)\right) .
$$

3.3. The method of good and bad historical paths. Fix $K \in \mathbf{K}^{\nu}$, for some $\nu>0$, and a finite measure $\mu$ on $\mathrm{R}^{d}$. Consider the historical SBM $\tilde{X}$ of Proposition 7 starting from $\tilde{X}_{0}=\mu$. First we introduce some Brownian stopping times and small constants.

Hypothesis 8 (Stage quantities). Let $0<\varepsilon<1$ and $N=N(\varepsilon) \geq 0$.

(a) (Stage duration) Consider Brownian stopping times

$$
0 \leq T_{N}^{\varepsilon}<T_{N+1}^{\varepsilon}<\cdots<T_{\infty}^{\varepsilon}<\infty,
$$

where the bound $T_{\infty}^{\varepsilon}$ is nonrandom.

(b) (Constants) For $n \geq N=N(\varepsilon)$, let $M_{n}^{\varepsilon}, \delta_{n}^{\varepsilon}, \lambda_{n}^{\varepsilon}, \xi_{n}^{\varepsilon}$ and, in addition, $\delta_{N-1}^{\varepsilon}$ be (strictly) positive constants with the following properties:

$$
\begin{gathered}
M_{n}^{\varepsilon} \downarrow 0 \quad \text { as } n \uparrow \infty . \\
\lim _{\varepsilon \downarrow 0}\left(\delta_{N-1}^{\varepsilon}+\sum_{n \geq N}\left(\delta_{n}^{\varepsilon}+\lambda_{n}^{\varepsilon}\right)\right)=0 .
\end{gathered}
$$

Introduce the set $E_{n}^{\varepsilon}$ of so-called good historical paths (during [ $\left.T_{n}^{\varepsilon}, T_{n+1}^{\varepsilon}\right]$ ),

$$
E_{n}^{\varepsilon}:=\left\{w \in \mathbf{C}: \int_{T_{n}^{\varepsilon}}^{T_{n+1}^{\varepsilon}} K(d r) \geq \xi_{n}^{\varepsilon}\right\},
$$

that is, paths $w$ with at least the amount $\xi_{n}^{\varepsilon}$ of accumulated branching over the time interval $\left[T_{n}^{\varepsilon}, T_{n+1}^{\varepsilon}\right)$. We call $\left(E_{n}^{\varepsilon}\right)^{\mathrm{c}}=\mathbf{C} \backslash E_{n}^{\varepsilon}$ the set of bad paths. On the good and bad paths we impose the following hypothesis.

HYPOTHESIS 9 (Good and bad paths). Fix $\varepsilon \in(0,1)$. First,

$$
\widetilde{P}_{0, \mu}\left(\left\|\tilde{X}_{T_{N}^{\varepsilon}}\right\|>M_{N}^{\varepsilon}\right) \leq \delta_{N-1}^{\varepsilon}
$$

and for all $n \geq N=N(\varepsilon)$,

$$
\begin{aligned}
& \widetilde{P}_{0, \mu}\left\{\widetilde{X}_{T_{n+1}^{\varepsilon}}\left(E_{n}^{\varepsilon}\right)>0 \mid\left\|\widetilde{X}_{T_{n}^{\varepsilon}}\right\| \leq M_{n}^{\varepsilon}\right\} \leq \delta_{n}^{\varepsilon}, \\
& \widetilde{P}_{0, \mu}\left\{\widetilde{X}_{T_{n+1}^{\varepsilon}}\left(\left(E_{n}^{\varepsilon}\right)^{\mathrm{c}}\right) \mid\left\|\widetilde{X}_{T_{n}^{\varepsilon}}\right\| \leq M_{n}^{\varepsilon}\right\} \leq \lambda_{n}^{\varepsilon} M_{n+1}^{\varepsilon}
\end{aligned}
$$

[recall convention (39)]. 
Here is our interpretation of Hypothesis 9. Recall that by Hypothesis 8(b) the numbers $M_{n}^{\varepsilon}, \delta_{N-1}^{\varepsilon}, \delta_{n}^{\varepsilon}$ and $\lambda_{n}^{\varepsilon}$ are small. So at the beginning of the $N$ th stage, the total mass $\left\|\widetilde{X}_{T_{N}^{\varepsilon}}\right\|$ is already small with a high $\widetilde{P}_{0, \mu}$-probability. Then starting with a small mass at the beginning of the $n$th stage, our condition (43) says that good paths survive only with a small (conditional) probability in the present stage, whereas (44) means that the (conditional) expected mass of bad paths is small.

Our abstract criterion now reads as follows. Recall that $d=1, \mu \in \mathscr{M}_{f}$, and that $K \in \mathbf{K}^{\nu}$ for some $\nu>0$.

THEOREM 10 (Abstract criterion for finite time extinction). Impose Hypotheses 8 and 9. Then with $\widetilde{P}_{0, \mu}$-probability $1, \widetilde{X}_{t}=0$ for some $t$.

We mention that under additional conditions, estimate (43) can be obtained by a comparison with Feller's branching diffusion; see Section 3.5 below, whereas the expectation formula for stopped historical SBM is available to reduce assertion (44) to a statement on the probability of a path to be bad, that is, to have a small accumulated rate of branching. In fact, by the special Markov property and the expectation formula (37) applied to the indicator function $\varphi=\mathbf{1}_{\mathrm{R}_{+} \times\left(E_{\eta}^{\varepsilon}\right)^{\mathrm{c}}}$ and the starting measure $\eta=\widetilde{X}_{T_{n}^{\varepsilon}}$, we have

$$
\widetilde{P}_{0, \mu}\left\{\widetilde{X}_{T_{n+1}^{\varepsilon}}\left(\left(E_{n}^{\varepsilon}\right)^{\mathrm{c}}\right) \mid \mathscr{G}_{T_{n}^{\varepsilon}}\right\}=\widetilde{\Pi}_{\widetilde{X}_{T_{n}^{\varepsilon}}}\left(\widetilde{W}_{T_{n+1}^{\varepsilon}} \in\left(E_{n}^{\varepsilon}\right)^{\mathrm{c}}\right) \text {. }
$$

By definition, and since $E_{n}^{\varepsilon}$ only depends on $\left\{w_{s}: T_{n}^{\varepsilon} \leq s \leq T_{n+1}^{\varepsilon}\right\}$, we can continue with

$$
\begin{aligned}
& =\int \tilde{X}_{T_{n}^{\varepsilon}}(d s, d w) \widetilde{\Pi}_{s, w}\left(\widetilde{W}_{T_{n+1}^{\varepsilon}} \in\left(E_{n}^{\varepsilon}\right)^{\mathrm{c}}\right) \\
& =\int \tilde{X}_{T_{n}^{\varepsilon}}(d s, d w) \Pi_{s, w_{s}}\left(W \in\left(E_{n}^{\varepsilon}\right)^{\mathrm{c}}\right) .
\end{aligned}
$$

Combining (45) and (46) implies the following result.

LEMMA 11 (Sufficient condition). If the estimate

$$
\Pi_{s, w_{s}}\left(W \in\left(E_{n}^{\varepsilon}\right)^{\mathrm{c}}\right) \leq \lambda_{n} \frac{M_{n+1}^{\varepsilon}}{M_{n}^{\varepsilon}}, \quad(s, w) \in \operatorname{supp} X_{T_{n}^{\varepsilon}},
$$

holds, then the conditional expectation estimate (44) is true.

3.4. Proof of the abstract criterion. Here we want to prove Theorem 10 . For $0<\varepsilon<1$ and $N=N(\varepsilon) \geq 0$, set

$$
A_{n}^{\varepsilon}:=\left\{\left\|\tilde{X}_{T_{n}^{\varepsilon}}\right\| \leq M_{n}^{\varepsilon}\right\}, \quad n \geq N \quad \text { and } \quad A^{\varepsilon}:=\bigcap_{n \geq N} A_{n}^{\varepsilon}
$$

as well as

$$
\bar{T}_{\infty}^{\varepsilon}:=\lim _{n \uparrow \infty} T_{n}^{\varepsilon} .
$$

Note that this limiting Brownian stopping time satifies $\bar{T}_{\infty}^{\varepsilon} \leq T_{\infty}^{\varepsilon}<\infty$. 
$1^{\prime}$ (Extinction on $\left.A^{\varepsilon}\right)$. First, we show that for all $\varepsilon \in(0,1)$,

$$
\left\|\widetilde{X}_{\bar{T}_{\infty}^{\varepsilon}}\right\|=0 \text { on } A^{\varepsilon}, \widetilde{P}_{0, \mu} \text {-a.s. }
$$

Indeed, fix $0<\varepsilon<1$ and a $\zeta>0$. Then by Markov's inequality, for each $n \geq N$,

$$
\widetilde{P}_{0, \mu}\left(\left\{\left\|\widetilde{X}_{\bar{T}_{\infty}^{\varepsilon}}\right\|>\zeta\right\} \cap A^{\varepsilon}\right) \leq \zeta^{-1} \widetilde{P}_{0, \mu} \mathbf{1}_{A_{n}^{\varepsilon}}\left\|\widetilde{X}_{\bar{T}_{\infty}^{\varepsilon}}\right\|
$$

However, by the special Markov property, the expectation formula (37) and the definition of $A_{n}^{\varepsilon}$,

$$
\widetilde{P}_{0, \mu}\left\{\mathbf{1}_{A_{n}^{\varepsilon}}\left\|\widetilde{X}_{\bar{T}_{\infty}^{\varepsilon}}\right\| \mid \mathscr{I}_{T_{n}^{\varepsilon}}\right\}=\mathbf{1}_{A_{n}^{\varepsilon}} \widetilde{P}_{\widetilde{X}_{T_{n}^{\varepsilon}}}\left\|\widetilde{X}_{\bar{T}_{\infty}^{\varepsilon}}\right\|=\mathbf{1}_{A_{n}^{\varepsilon}}\left\|\widetilde{X}_{T_{n}^{\varepsilon}}\right\| \leq M_{n}^{\varepsilon} .
$$

Hence, estimate (51) can be continued with

$$
\leq \zeta^{-1} M_{n}^{\varepsilon} \underset{n \uparrow \infty}{\longrightarrow} 0
$$

by Hypothesis 8(b1). Thus,

$$
\widetilde{P}_{0, \mu}\left(\left\{\left\|\tilde{X}_{\bar{T}_{\infty}^{\varepsilon}}\right\|>\zeta\right\} \cap A^{\varepsilon}\right)=0 \quad \forall \zeta>0
$$

and (50) follows.

$2^{\prime}$ ( $A_{n+1}^{\varepsilon}$ fails with small conditional probability). From Markov's inequality and the conditional expectation estimate (44) we get, for $0<\varepsilon<1$ and $n \geq N$,

$$
\widetilde{P}_{0, \mu}\left\{\widetilde{X}_{T_{n+1}^{\varepsilon}}\left(\left(E_{n}^{\varepsilon}\right)^{\mathrm{c}}\right)>M_{n+1}^{\varepsilon} \mid A_{n}^{\varepsilon}\right\} \leq \lambda_{n}^{\varepsilon} .
$$

Together with (43), we conclude for

$$
\widetilde{P}_{0, \mu}\left\{\left(A_{n+1}^{\varepsilon}\right)^{\mathrm{c}} \mid A_{n}^{\varepsilon}\right\} \leq \delta_{n}^{\varepsilon}+\lambda_{n}^{\varepsilon} .
$$

$3^{\prime}$ ( $A^{\varepsilon}$ fails with small probability). Next we show that

$$
\lim _{\varepsilon \downarrow 0} \widetilde{P}_{0, \mu}\left(\left(A^{\varepsilon}\right)^{\mathrm{c}}\right)=0 .
$$

We decompose the complement $\left(A^{\varepsilon}\right)^{\mathrm{c}}$ of $A^{\varepsilon}$ according to the smallest natural number $n \geq N$ such that $A_{n}^{\varepsilon}$ fails:

$$
\begin{aligned}
\widetilde{P}_{0, \mu}\left(\left(A^{\varepsilon}\right)^{\mathrm{c}}\right) & =\widetilde{P}_{0, \mu}\left(\left(A_{N}^{\varepsilon}\right)^{\mathrm{c}}\right)+\sum_{n \geq N} \widetilde{P}_{0, \mu}\left(A_{N}^{\varepsilon} \cap \cdots \cap A_{n}^{\varepsilon} \cap\left(A_{n+1}^{\varepsilon}\right)^{\mathrm{c}}\right) \\
& \leq \widetilde{P}_{0, \mu}\left(\left(A_{N}^{\varepsilon}\right)^{\mathrm{c}}\right)+\sum_{n \geq N} \widetilde{P}_{0, \mu}\left\{\left(A_{n+1}^{\varepsilon}\right)^{\mathrm{c}} \mid A_{n}^{\varepsilon}\right\} .
\end{aligned}
$$

Then (56) follows from (42), step $2^{\prime}$ and Hypothesis 8(b2). 
$4^{\prime}$ (Conclusion). Let $\varepsilon=k^{-1}, k>1$. From (56) and the monotonicity property of measures, we learn that the event that $A^{1 / k}$ fails for all $k$, has $\widetilde{P}_{0, \mu^{-}}$probability 0 . In other words,

$$
\widetilde{P}_{0, \mu}\left(\bigcup_{k>1} A^{1 / k}\right)=1 .
$$

Then step $1^{\prime}$ implies that

$$
\exists k>1 \text { such that } \tilde{X}_{T_{\infty}^{1 / k}}=0, \quad \widetilde{P}_{0, \mu} \text {-a.s. }
$$

Again applying the special Markov property, we obtain

$$
\exists k \text { such that } \tilde{X}_{T_{\infty}^{1 / k}}=0, \quad \widetilde{P}_{0, \mu} \text {-a.s. }
$$

Since $T_{\infty}^{1 / k}$ is nonrandom, the proof of our abstract Theorem 10 is finished.

3.5. Scaled comparison with Feller's branching diffusion. Consider a pair of Brownian stopping times $0 \leq T_{0}<T_{1}$, a constant $\xi>0$, and define the set of good historical paths

$$
E:=\left\{w \in \mathbf{C}: \int_{T_{0}}^{T_{1}} K(d r) \geq \xi\right\} .
$$

We want to estimate a conditional probability as in (43) of Hypothesis 9 under a mild additional assumption on the branching rate functional $K$. For this purpose, we will compare with the survival probability in Feller's branching diffusion.

Recall that $d \geq 1, \mu \in \mathscr{M}_{f}$, and that $\mathscr{G}_{T_{0}}$ denotes the pre- $T_{0} \sigma$-field.

Proposition 12 (Comparison with Feller's branching diffusion). Assume that the branching rate functional $K \in \mathbf{K}^{\nu}(\nu>0)$ is homogeneous and satisfies

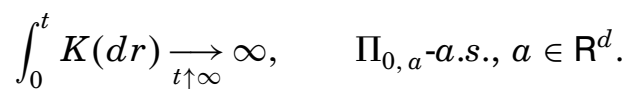

Then $\widetilde{P}_{0, \mu}$-almost surely,

$$
\widetilde{P}_{0, \mu}\left\{\widetilde{X}_{T_{1}}(E)>0 \mid \mathscr{G}_{T_{0}}\right\} \leq \frac{1}{\xi}\left\|\widetilde{X}_{T_{0}}\right\| .
$$

We will prove Proposition 12 in the next subsection, using an idea from [20], which was in turn inspired by a modulus of continuity technique of [10]. In fact, since the paths in $E$ have a "significant" accumulated rate of branching $\xi$ over the time interval $\left[T_{0}, T_{1}\right.$ ) [recall (41)], we can compare (in law) $\widetilde{X}_{T_{1}}(E)$ with the mass in Feller's branching diffusion after an appropriate individual time change. (Recall that the total mass of the classical super-Brownian motion is equal in distribution to Feller's branching diffusion.) But for Feller's branching diffusion, there is a well-known formula for the probability that the process survives by a given time. 
REMARK 13. We usually apply Proposition 12 for $T_{0}=T_{n}^{\varepsilon}, T_{1}=T_{n+1}^{\varepsilon}$, $\xi=\xi_{n}^{\varepsilon}$ and $E=E_{n}^{\varepsilon}$, with fixed $\varepsilon$ and $n$.

3.6. Proof of the comparison argument. Consider the process $\left\{\tilde{X}_{t}: t \geq T_{0}\right\}$, given $\mathscr{I}_{T_{0}}$. In particular, the starting measure $\tilde{X}_{T_{0}}=: \vartheta$ is given. Note that this (conditional) process has the law $\widetilde{P}_{\vartheta}$, by the special Markov property. In order to prepare for the proof of Proposition 12, we first intend to define a new time scale denoted by $r$, dictated by the additive functional $K$. Given for the moment a path $w \in \mathbf{C}$, set

$$
R(t):=\int_{T_{0}}^{T_{0}+t} K(d s), \quad t \geq 0
$$

(recall that $K$ is a continuous additive functional of Brownian motion $W$ ). Note that $R(t) \uparrow \infty$ as $t \uparrow \infty, \widetilde{\Pi}_{\vartheta}$-almost everywhere [by assumption (62)] and that $R(t)$ depends continuously on $t$ [by the continuity of $K$ ]. Define finite (Brownian) stopping times $\tau(r)$ (converging to infinity as $r \uparrow \infty$ ) by

$$
\tau(r):=\inf \{t>0, R(t) \geq r\}, \quad r \geq 0 .
$$

Consider the stopped historical SBM $r \mapsto \widetilde{X}_{T_{0}+\tau(r)}$. Put

$$
Z_{r}:=\left\|\tilde{X}_{T_{0}+\tau(r)}\right\|, \quad r \geq 0,
$$

for its total mass process. This leads us to Feller's branching diffusion (without drift).

LEMMA 14 ("Embedded" Feller's branching diffusion). $\widetilde{P}_{0, \mu}$-a.s., under the probability laws $\widetilde{P}_{\vartheta}$ with $\vartheta=\widetilde{X}_{T_{0}}$, the process $r \mapsto Z_{r}$ satisfies equation (7) with $Z_{0}=\|\vartheta\|$.

Proof. The initial condition is trivially fulfilled. To simplify notation, we write $\mathscr{G}_{r}$ for the pre- $\left[T_{0}+\tau(r)\right] \sigma$-field. It is sufficient to show that $\widetilde{P}_{0, \mu}$-almost surely, $Z$ is a $\left(\widetilde{P}_{\vartheta},\left(\mathscr{G}_{r}\right)_{r \geq 0}\right)$-martingale with square variation

$$
\langle\langle Z\rangle\rangle_{r}=2 \int_{0}^{r} d s Z_{s}, \quad r \geq 0 .
$$

This would be verified if we proved that for $0 \leq r<r^{\prime}$,

$$
\begin{aligned}
\widetilde{P}_{\vartheta}\left\{Z_{r^{\prime}} \mid \mathscr{G}_{r}\right\} & =Z_{r}, \\
\widetilde{P}_{\vartheta}\left\{Z_{r^{\prime}}^{2}-2 \int_{r}^{r^{\prime}} d s Z_{s} \mid \mathscr{G}_{r}\right\} & =Z_{r}^{2} .
\end{aligned}
$$

By the special Markov property, statement (68) follows from the expectation formula (37). But then (69) reduces to

$$
\tilde{\operatorname{Var}}{ }_{\vartheta(r)} Z_{r^{\prime}}=2\left(r-r^{\prime}\right) Z_{r}
$$


with $\vartheta(r):=\widetilde{X}_{T_{0}+\tau(r)}$. But from the variance formula (38), the left-hand side of (70) equals

$$
2 \widetilde{\Pi}_{\vartheta}(r) \int_{\tau(r)}^{\tau\left(r^{\prime}\right)} K(d s)
$$

Using the definition (65) of $\tau(r)$, by a change of variables; see, for example, [30], Proposition (0.4.9), we can continue with

$$
=2 \widetilde{\Pi}_{\vartheta(r)} \int_{r}^{r^{\prime}} d s=2\left(r^{\prime}-r\right)\|\vartheta(r)\|
$$

getting the right-hand side of (70). This completes the proof.

Completion of Proof of Proposition 12. Recall that the left-hand side in $(63)$ can be written as $\widetilde{P}_{\vartheta}\left(\widetilde{X}_{T_{1}}(E)>0\right)$ with $\vartheta=\widetilde{X}_{T_{0}}$. Consider the event $\widetilde{X}_{T_{1}}(E)>0$. This means that at time $T_{1}$ there are historical paths alive which have at least an accumulated rate of branching of $\xi$. Trivially, these paths must be alive in the moment $T_{0}+\tau(\xi)$ when the accumulated rate of branching just reaches $\xi$. Consequently, $Z_{\xi}=\left\|\widetilde{X}_{T_{0}+\tau(\xi)}\right\|>0$. But by the embedded Feller's branching diffusion according to Lemma 14,

$$
\widetilde{P}_{\vartheta}\left(Z_{\xi}>0\right)=1-\exp \left[-\xi^{-1}\|\vartheta\|\right] \leq \xi^{-1}\|\vartheta\|
$$

and the claim (63) follows.

4. Two applications of the abstract criterion. Here we want to apply our abstract finite time extinction criterion, combined with the comparison with Feller's branching diffusion, to two one-dimensional models, namely SBM with the power law catalyst $\chi_{q}$ of Model 3, and with a certain point catalyst $\underline{\Gamma}$ with atoms whose locations are dense in $\mathrm{R}$ (we will need $\underline{\Gamma}$ later on).

To contrast our results, we will add also two "counterexamples", that is, nontrivial examples of catalytic super-Brownian motions where finite time extinction is violated; see Section 4.3 below.

4.1. Power law catalyst $\chi_{q}$ (Model 3). In the power law catalyst model, the branching rate functional $K$ is given by the BCLT $L_{[W, \psi]}$ (recall Lemma 4 ). Here the measure $\psi(d b) \in \mathscr{M}_{p}, \quad p>1$, has a density function $\chi_{q}(b)=|b|^{q} \wedge 1$, $b \in \mathrm{R}$, with respect to the (normalized) Lebesgue measure $d b$, as introduced in (9) (where the exponent $q>0$ is a fixed constant).

Since the catalyst is small only near the origin $b=0$, the bad historical paths will be those which spend a large amount of time near 0 . To estimate the probability of such paths, we need the following lemma. Let $L_{t}(b)$ denote the local time at $b \in \mathrm{R}$ of a (one-dimensional standard) Brownian path $W$, up to time $t$. 
LEMMA 15 (Brownian local time large deviations I). There exists a constant $c_{0}>0$ such that for all $\theta \in(0,1]$, and for all $a \in \mathrm{R}$, the following holds:

$$
\Pi_{0, a}\left(\int_{-\theta}^{\theta} d b L_{t}(b) \geq \frac{t}{2}\right) \leq \exp \left[-\frac{c_{0} t}{\theta^{2}}\right], \quad t \geq 0 .
$$

Proof. By Brownian scaling, we have

$$
\Pi_{0, a}\left(\int_{-\theta}^{\theta} d b L_{t}(b) \geq \frac{t}{2}\right)=\Pi_{0, a / \theta}\left(\int_{-1}^{1} d b L_{t / \theta^{2}}(b) \geq \frac{t}{2 \theta^{2}}\right) .
$$

So it suffices to prove the claim for $\theta=1$. But then we may apply Lemma 2.2 of Donsker and Varadhan [12] with $A$ there the set of subprobability measures $\nu$ on $\mathrm{R}$ such that $\nu([-1,1]) \geq 1 / 2$.

In order to specify the quantities entering in Hypotheses 8 and 9, fix a constant $\alpha>0$ and a $\beta \in(0,2 \alpha)$. For $n \geq N(\varepsilon) \equiv 0$, and $0<\varepsilon<1$, put

$$
\begin{gathered}
\theta_{n}:=e^{-\alpha n}, \quad M_{n}^{\varepsilon} \equiv M_{n}:=\exp (-(1+\beta+\alpha q) n), \\
t_{n}^{\varepsilon}:=\varepsilon^{-1} e^{-\beta n}, \quad \xi_{n}^{\varepsilon}:=\frac{1}{2} t_{n}^{\varepsilon} \theta_{n}^{q}, \quad \delta_{n}^{\varepsilon}:=M_{n} / \xi_{n}^{\varepsilon}, \quad \delta_{-1}^{\varepsilon}:=\varepsilon,
\end{gathered}
$$

and finally,

$$
\lambda_{n}^{\varepsilon}:=\frac{M_{n}}{M_{n+1}} \exp \left[-\frac{c_{0}}{\theta_{n}^{2}} t_{n}^{\varepsilon}\right]
$$

(with $c_{0}$ from Lemma 15). We will use the deterministic times

$$
T_{n+1}^{\varepsilon}:=T_{n}^{\varepsilon}+t_{n}^{\varepsilon}, \quad T_{0}^{\varepsilon}:=0 .
$$

By Remark 1 we may assume without loss of generality that $\mu(\mathrm{R}) \leq 1$. Then the "starting condition" (42) is trivially satisfied.

Note that these constants satisfy Hypothesis 8. In fact, the series in condition (b2) can be estimated from above by

$$
c \sum_{n \geq 0}\left[\varepsilon e^{-n}+\exp \left[-c_{0} \varepsilon^{-1} \exp ((2 \alpha-\beta) n)\right]\right] .
$$

Since $e^{(2 \alpha-\beta) n} \geq c n$, this bond is of order $\varepsilon$, and property (b2) follows.

By the choice of $\delta_{n}^{\varepsilon}$, inequality (43) concerning the good paths holds by the comparison Proposition 12.

It remains to verify the conditional expectation estimate (44) for the mass of bad paths at time $T_{n-1}^{\varepsilon}$, for which we will use Lemma 11. By time-homogeneity and definition (41) of $E_{n}^{\varepsilon}$, for $s=T_{n}^{\varepsilon}$ and $a \in \mathrm{R}$,

$$
\Pi_{s, a}\left(W \in\left(E_{n}^{\varepsilon}\right)^{\mathrm{c}}\right)=\Pi_{0, a}\left(\int_{0}^{t_{n}^{\varepsilon}} K(d r) \leq \xi_{n}^{\varepsilon}\right)
$$

and

$$
\int_{0}^{t_{n}^{\varepsilon}} K(d r)=\int_{0}^{t_{n}^{\varepsilon}} d r \chi_{q}\left(W_{r}\right)=\int d b \chi_{q}(b) L_{t_{n}^{\varepsilon}}(b) \geq \int_{|b| \geq \theta_{n}} d b \theta_{n}^{q} L_{t_{n}^{\varepsilon}}(b) .
$$


Thus, the probability expression in (81) can be estimated from above by

$$
\Pi_{0, a}\left(\int_{|b| \geq \theta_{n}} d b L_{t_{n}^{\varepsilon}}(b) \leq \frac{t_{n}^{\varepsilon}}{2}\right)=\Pi_{0, a}\left(\int_{|b| \leq \theta_{n}} d b L_{t_{n}^{\varepsilon}}(b) \geq \frac{t_{n}^{\varepsilon}}{2}\right) .
$$

Hence, by Lemma 15, we get

$$
\Pi_{T_{n}^{\varepsilon}, a}\left(W \in\left(E_{n}^{\varepsilon}\right)^{\mathrm{c}}\right) \leq \exp \left[-\frac{c_{0}}{\theta_{n}^{2}} t_{n}^{\varepsilon}\right]=\frac{M_{n+1}}{M_{n}} \lambda_{n}^{\varepsilon},
$$

where we used (78). In other words, (47) in Lemma 11 holds, and (44) is valid.

Altogether, we showed that all requirements for the abstract criterion Theorem 10 are satisfied, hence finite time extinction holds for the SBM with power law catalyst $\chi_{q}$ for any finite initial measure $\mu$ on $\mathrm{R}$.

It is reasonable to expect that finite time extinction will fail if we replace catalyst's depression at 0 by a still deeper one with an adequate exponential decay instead of the power law; see Corollary 17(i) below.

4.2. A point catalyst with dense locations. Now we consider the case

$$
K=L_{[W, \underline{\Gamma}]} \quad \text { with } \underline{\Gamma}=\sum_{n=N}^{\infty} 2^{-n} \underline{\pi}_{n}
$$

for a fixed $N \geq 0$, independent of $\varepsilon$. Here $\underline{\pi}_{n}$ is assumed to be a (two-sided infinite, locally finite, deterministic) point measure on $\mathrm{R}$ such that all neighboring points have a distance of at most $\Delta_{n}$, where, for some $\beta \in(0,1)$,

$$
\Delta_{n}:=e^{-\beta n}, \quad n \geq N \text {. }
$$

We claim that the continuous SBM $X^{\Gamma}$ with catalyst $\underline{\Gamma}$ has the finite time extinction property. This will follow from our abstract extinction criterion Theorem 10 once we have found the appropriate quantities $T_{n}^{\varepsilon}, M_{n}^{\varepsilon}, \delta_{n}^{\varepsilon}, \lambda_{n}^{\varepsilon}, \xi_{n}^{\varepsilon}$ entering into Hypotheses 8 and 9.

$1^{\prime}$ (Some constants). Choose $\alpha \in(\beta / 2, \beta)$. For $n \geq N$ and $0<\varepsilon<1$, set

$$
m_{n}^{\varepsilon}:=\left[\frac{e^{\alpha n}}{\varepsilon}\right], \quad s_{n}^{\varepsilon}:=\frac{e^{-\beta n}}{\varepsilon^{2}}, \quad t_{n}^{\varepsilon}:=2 m_{n}^{\varepsilon} s_{n}^{\varepsilon}, \quad M_{n}^{\varepsilon} \equiv M_{n}:=2^{-n}
$$

(where $[z]$ denotes the integer part of $z$ ). We again use deterministic times $T_{n+1}^{\varepsilon}:=T_{n}^{\varepsilon}+t_{n}^{\varepsilon}, T_{N}^{\varepsilon}:=0$. Note that by our choice of $t_{n}$ they satisfy (40). The quantities $\xi_{n}^{\varepsilon}, \delta_{n}^{\varepsilon}, \lambda_{n}^{\varepsilon}$ will be defined in (100), (101) and (107), respectively.

Assume without loss of generality that $\mu(\mathrm{R}) \leq 2^{-N}$. Then, if we set $\delta_{N-1}^{\varepsilon}=\varepsilon$, the starting condition (42) is trivially satisfied.

$2^{\prime}$ (Partitioning). For $n \geq N$ and $0<\varepsilon<1$ fixed, our next aim is to introduce a partition of the time period $\left[T_{n}^{\varepsilon}, T_{n+1}^{\varepsilon}\right)$ by means of some Brownian stopping times. This construction allows us to consider hitting times of neighboring points of $\underline{\pi}_{n}$ and local times spent on them.

Given $\vartheta_{n}:=\widetilde{X}_{T_{n}^{\varepsilon}}$ (which we do not need to consider as a stopped measure since $T_{n}^{\varepsilon}$ is deterministic), and a path $w$ "distributed" according to $\vartheta_{n}(d w)$, 
we consider the Brownian path process $\widetilde{W}$ distributed according to $\widetilde{\Pi}_{T_{n}^{\varepsilon}, w}$, and its projection $t \mapsto\left(\widetilde{W}_{t}\right)_{t}=W_{t}$ with law

$$
\Pi_{T_{n}^{\varepsilon}, w\left(T_{n}^{\varepsilon}\right)}=: \underline{\Pi} .
$$

(For typographical simplicity, sometimes we write $w(t)$ instead of $w_{t}$, etc.)

Set $\kappa_{0}:=T_{n}^{\varepsilon}$. For $m \geq 1$, we inductively define (Brownian) stopping times $\bar{\kappa}_{m}=\bar{\kappa}_{m, n}^{\varepsilon}(W)$ and $\kappa_{m}=\kappa_{m, n}^{\varepsilon}(W)$ as follows. Given $\kappa_{m-1}$, let $\bar{\kappa}_{m}$ denote the first time point $t \geq \kappa_{m-1}$ such that $W$ hits one of the atoms of $\underline{\pi}_{n}$. Given $\bar{\kappa}_{m}$, we simply define $\kappa_{m}=\bar{\kappa}_{m}+s_{n}^{\varepsilon}$ [with $s_{n}^{\varepsilon}>0$ introduced in (86)].

Write $H_{m}=H_{m, n}^{\varepsilon}$ for the hitting time $\bar{\kappa}_{m}-\kappa_{m-1}$ of $\underline{\pi}_{n}$ (starting at time $\left.\kappa_{m-1}\right)$. Recall that by definition the distance between neighboring atoms of $\underline{\pi}_{n}$ is at most $\Delta_{n}$. Let $H$ denote the first time $t$ that $\left|W_{t}\right|=\Delta_{n}$. Set $f(t, x):=$ $\Pi_{0, x}(H \geq t), t>0$. Then

$$
\underline{\Pi}\left(H_{m} \geq s_{n}^{\varepsilon}\right) \leq f\left(s_{n}^{\varepsilon}, 0\right) .
$$

But $f$ satisfies the heat equation

$$
\frac{\partial f}{\partial t}=\frac{1}{2} \Delta f \quad \text { on }(0, \infty) \times\left(-\Delta_{n}, \Delta_{n}\right)
$$

with initial condition $f(0, \cdot)=1$ and Dirichlet boundary condition $f\left(\cdot,-\Delta_{n}+\right)=$ $f\left(\cdot, \Delta_{n}-\right)=0$. Then (see, for example, [13], Section 1.8.4) we get the following eigenfunction representation

$$
f(t, x)=\sum_{k=1}^{\infty} \underline{c}_{k} \cos \left(\frac{k \pi x}{\Delta_{n}}\right) \exp \left[\frac{-k^{2} \pi^{2} t}{\Delta_{n}^{2}}\right],
$$

where the constants $\underline{c}_{k}$ satisfy $\sum_{k=1}^{\infty}\left|\underline{c}_{k}\right|<\infty$. Thus

$$
f(t, 0) \leq c_{0}^{-1} \exp \left[\frac{-c_{0} t}{\Delta_{n}^{2}}\right], \quad t>0,
$$

for some constant $c_{0}>0$. Hence, together with (88),

$$
\underline{\Pi}\left(H_{m} \geq s_{n}^{\varepsilon}\right) \leq c_{0}^{-1} \exp \left[-\frac{c_{0} s_{n}^{\varepsilon}}{\Delta_{n}^{2}}\right], \quad m \geq 0, n \geq N .
$$

Therefore,

$$
\underline{\Pi}\left(\sum_{m=1}^{m_{n}^{\varepsilon}} H_{m} \geq m_{n}^{\varepsilon} s_{n}^{\varepsilon}\right) \leq c_{0}^{-1} m_{n}^{\varepsilon} \exp \left[-\frac{c_{0} s_{n}^{\varepsilon}}{\Delta_{n}^{2}}\right]=: \zeta_{n}^{\varepsilon}
$$

[with $\Delta_{n}, s_{n}^{\varepsilon}, m_{n}^{\varepsilon}$ introduced in (85) and (86)].

Now write $L_{m}=L_{m, n}^{\varepsilon}$ for the (Brownian) local time spent by $W$ at the site $W\left(\bar{\kappa}_{m}\right)$ during the time interval $\left[\bar{\kappa}_{m}, \kappa_{m}\right)$ of length $s_{n}^{\varepsilon}$. That is, symbolically,

$$
L_{m}:=\int_{\bar{\kappa}_{m}}^{\kappa_{m}} d r \delta_{W\left(\bar{\kappa}_{m}\right)}\left(W_{r}\right) \text {. }
$$


Recall that at this site $W\left(\bar{\kappa}_{m}\right)$ there is an atom of $\underline{\pi}_{n}$, and that the mass $2^{-n}$ is attached to it. Therefore, using the integers $m_{n}^{\varepsilon}$ introduced in (86), for the BCLT $L_{[W, \underline{\Gamma}]}$ of $\underline{\Gamma} \geq 2^{-n} \underline{\pi}_{n}$ we get

$$
\int_{T_{n}^{\varepsilon}}^{\kappa_{m_{n}^{\varepsilon}}} L_{[W, \underline{\Gamma}]}(d r) \geq 2^{-n} \sum_{m=1}^{m_{n}^{\varepsilon}} L_{m} .
$$

Clearly, the $L_{m}$ are i.i.d. (with respect to $\underline{\Pi}$ ). Moreover, $L_{m}$ is equal in law to (96)

$$
\sup _{0 \leq t \leq s_{n}^{\varepsilon}} W_{t}^{0},
$$

where $W^{0}$ is distributed according to $\Pi_{0,0}$ (see, e.g., [30], Theorem (6.2.3)). Scaling time, we find that $\left(s_{n}^{\varepsilon}\right)^{-1 / 2} L_{m}$ is equal in law to

$$
L^{0}:=\sup _{0 \leq t \leq 1} W_{t}^{0}
$$

(which is independent of $n$ and $\varepsilon$ ). Set $a:=\frac{1}{2} \Pi_{0,0} L^{0}$. Since $L^{0}$ has finite exponential moments, by standard large deviation estimates (see, e.g., [2], Theorem 9.3) there exists a constant $c_{1}>0$ such that

$$
\underline{\Pi}\left(\left(s_{n}^{\varepsilon}\right)^{-1 / 2} \sum_{m=1}^{k} L_{m}<a k\right) \leq \exp \left(-2 c_{1} k\right), \quad k \geq 1 .
$$

Combining with (95), we thus have

$$
\underline{\Pi}\left(\int_{T_{n}^{\varepsilon}}^{\kappa_{m_{n}^{\varepsilon}}} L_{[W, \underline{\Gamma}]}(d r)<\xi_{n}^{\varepsilon}\right) \leq \exp \left[-2 c_{1} m_{n}^{\varepsilon}\right]
$$

where

$$
\xi_{n}^{\varepsilon}:=a m_{n}^{\varepsilon}\left(s_{n}^{\varepsilon}\right)^{1 / 2} 2^{-n} .
$$

$3^{\prime}$ (Good and bad historical paths). Recall the set $E_{n}^{\varepsilon}$ of good paths introduced in formula line (41) [based on $t_{n}^{\varepsilon}$ defined in (86) and entering into (79), as well as $\xi_{n}^{\varepsilon}$ from (100)]. Since the BCLT $L_{[W, \Gamma]}$ satisfies (62), by Proposition 12 we get the survival probability estimate (43) for the good paths, if we set

$$
\delta_{n}^{\varepsilon}:=M_{n} / \xi_{n}^{\varepsilon} .
$$

On the other hand, in order to calculate the expected mass of bad paths as required in (44), we look at

$$
\underline{\Pi}\left(\left(E_{n}^{\varepsilon}\right)^{\mathrm{c}}\right) \text {. }
$$

In order to further estimate this, consider two cases. First let $w$ have "large" hitting times, that is,

$$
\sum_{m=1}^{m_{n}^{\varepsilon}} H_{m} \geq m_{n}^{\varepsilon} s_{n}^{\varepsilon}
$$


By (93), this occurs with a $\underline{\Pi}$-probability bounded by $\zeta_{n}^{\varepsilon}$. In the opposite case of (103), by the definition of $T_{n+1}^{\varepsilon}$ we have

$$
\kappa_{m_{n}^{\varepsilon}}=T_{n}^{\varepsilon}+m_{n}^{\varepsilon} s_{n}^{\varepsilon}+\sum_{m=1}^{m_{n}^{\varepsilon}} H_{m}<T_{n+1}^{\varepsilon}
$$

[recall (86) and (79)]. Hence here $W \in\left(E_{n}^{\varepsilon}\right)^{\mathrm{c}}$ implies, by the definition (41) of $E_{n}^{\varepsilon}$, that

$$
\int_{T_{n}^{\varepsilon}}^{\kappa_{m_{n}^{\varepsilon}}^{\varepsilon}} L_{[W, \underline{\Gamma}]}(d r)<\xi_{n}^{\varepsilon}
$$

The II-probability of this event is estimated in (99). Altogether, for (102) we get the bound

$$
\zeta_{n}^{\varepsilon}+\exp \left[-2 c_{1} m_{n}^{\varepsilon}\right]
$$

[with $\zeta_{n}^{\varepsilon}$ from (93)]. If we now set

$$
\lambda_{n}^{\varepsilon}:=\frac{M_{n}}{M_{n+1}}\left[\zeta_{n}^{\varepsilon}+\exp \left[-2 c_{1} m_{n}^{\varepsilon}\right]\right]
$$

we obtain (47) in Lemma 11 which gives (44).

$4^{\prime}$ (Verification of the stage quantities). It remains to check that $\delta_{n}^{\varepsilon}$ and $\lambda_{n}^{\varepsilon}$ introduced in (101) and (107), respectively, satisfy Hypothesis 8(b2). First, by (101) and (100), $\delta_{n}^{\varepsilon}$ approximately equals

$$
c \varepsilon^{2} \exp \left[-\left(\alpha-\frac{\beta}{2}\right) n\right]
$$

hence its sum over $n$ is of order $\varepsilon^{2}$. Next, since $m_{n}^{\varepsilon} \geq c \varepsilon^{-1} n$, the second term of $\lambda_{n}^{\varepsilon}$ is bounded by $\exp \left[-c \varepsilon^{-1} n\right]$, except a constant factor. Summing over $n$ we arrive at a term of order $\varepsilon$. Finally, by (93), the first term of $\lambda_{n}^{\varepsilon}$ is bounded from above by

$$
c_{0}^{-1} \varepsilon^{-1} \exp \left[\alpha n-c_{0} \varepsilon^{-2} e^{\beta n}\right] .
$$

But

$$
\varepsilon^{-1} \int_{1}^{\infty} d x \exp \left[\alpha x-c_{0} \varepsilon^{-2} e^{\beta x}\right] \leq(\beta \varepsilon)^{-1} \int_{1}^{\infty} d y y^{\frac{\alpha}{\beta}-1} \exp \left[-c_{0} \varepsilon^{-2} y\right],
$$

and $y^{\frac{\alpha}{\beta}-1} \leq 1$ by our assumptions on $\alpha, \beta, y$. Hence, it suffices to consider

$$
\varepsilon^{-1} \int_{1}^{\infty} d y \exp \left[-c_{0} \varepsilon^{-2} y\right]
$$

which is of order $\varepsilon$. Consequently, the series in Hypothesis 8(b2) is bounded by $c \varepsilon$, hence this hypothesis is satisfied in the present case.

Summarizing, the catalytic $S B M X^{\Gamma}$ dies in finite time, for any finite starting measure $\mu$ on $\mathrm{R}$. 
4.3. Nontrivial examples for finite time survival. In this subsection we present the announced counterexamples for finite time extinction of superBrownian motion in $\mathrm{R}$ with some nontrivial catalysts. For simplicity, we always start with a unit mass at zero.

Recalling Lemma 4, consider the SBM $X=X^{\psi}$ with catalyst $\psi \in \mathscr{M}_{p}$, that is, with branching rate functional given by the Brownian collision local time $L_{[W, \psi]}$.

Proposition 16 (Finite time survival). Assume that the (random) catalyst $\psi$ in $\mathscr{M}_{p}$ satisfies the following condition: for all $K \geq 1$,

$$
\lim _{\varepsilon \downarrow 0} \frac{\psi((-\varepsilon, \varepsilon))}{\exp \left[-K / \varepsilon^{2}\right]}=0 \quad \text { a.s. }
$$

and for all Borel subsets $\mathscr{g}$ of $\mathrm{R}$ of Lebesgue measure zero,

$$
\psi(\mathscr{g})=0 \quad \text { a.s. }
$$

Then, for almost all realizations $\psi$ of the catalyst, the $S B M X=X^{\psi}$ survives for all finite times,

$$
P_{0, \delta_{0}}\left(X_{t}^{\psi} \neq 0\right)=1, \quad t \geq 0
$$

Roughly speaking, if the catalyst $\psi$ has a "superexponential depression" at 0 in the sense of (111), then in particular the related catalytic SBM $X^{\psi}$ cannot die in finite time. The proof of this proposition is postponed to the end of this subsection.

Corollary 17 (Special cases). The SBMs with the following catalysts $\psi$ survive for all finite times.

(i) (Absolutely continuous catalyst with superexponential depression.) Let $\psi \in \mathscr{M}_{p}$ be deterministic and have a bounded density function also denoted by $\psi$ which is strictly positive on $\mathrm{R} \backslash\{0\}$ and which satisfies

$$
\lim _{b \rightarrow 0} \frac{\psi(|b|)}{\exp \left[-K /|b|^{2}\right]}=0, \quad k>0 .
$$

(ii) (Catalyst with dense and rarified atoms.) Let $\psi$ be the homogeneous random measure on $\mathrm{R}$ with independent increments which has no deterministic part and the Lévy measure

$$
\nu(d b):=\mathbf{1}_{(0,1 / 2)}(b) \frac{1}{b \log (1 / b)} d b .
$$

PROOF. We will verify the assumptions in Proposition 16. Since part (i) is trivial, we only deal with (ii). First of all, note that $\nu((0,1 / 2))=\infty$, hence $\psi$ has atoms which are dense in R. On the other hand, the support of $\psi$ is countable. Furthermore, its law is shift invariant. Thus, with probability 1, 
this support does not hit a fixed null set $\mathscr{g}$ as in the proposition, that is, (112) holds. To show that $\psi$ satisfies (111) almost surely, it suffices to verify

$$
\limsup _{\varepsilon \downarrow 0} \frac{\psi((-\varepsilon, \varepsilon))}{\exp \left[-K / \varepsilon^{2}\right]}<\infty \quad \text { a.s., } \quad K \geq 1,
$$

since $K \geq 1$ is arbitrary in both statements. For fixed $K \geq 1$, set $h(\varepsilon):=$ $\exp \left[-K / \varepsilon^{2}\right]$. By Theorem 1 of [22], it is enough to show that for the inverse function $h^{-1}$ of $h$,

$$
\int_{0}^{1 / 2} \nu(d b) h^{-1}(b)<\infty
$$

But

$$
h^{-1}(b)=\frac{\sqrt{K}}{\sqrt{\log (1 / b)}}
$$

and

$$
\int_{0}^{1 / 2} d b \frac{1}{b(\log (1 / b))^{3 / 2}}<\infty
$$

completing the proof.

To prepare for the proof of Proposition 16, we first introduce another result on the Brownian local time $L_{t}(b)$ (at $b$ up to time $t$ ). For $\varepsilon>0$, let $\tau_{\varepsilon}=$ $\tau_{\varepsilon}(W)$ denote the first time that Brownian motion $W$ (with law $\Pi_{0,0}$ ) hits the boundary of the interval $(-\varepsilon, \varepsilon)$.

LEMMA 18 (Brownian local time large deviations II). There exist constants $c_{0}>0$ and $T>0$ such that

$$
\Pi_{0,0}\left(\sup _{b \in \mathrm{R}} L_{t}(b) \leq \frac{c_{0} t}{\varepsilon}, \tau_{\varepsilon}>t\right) \geq \exp \left[-\frac{c_{0} t}{\varepsilon^{2}}\right], \quad t \geq T, 0<\varepsilon \leq 1 .
$$

Proof. For $t>0$ fixed, we will consider $t^{-1} L_{t}$ as a probability measure on R. (Note that in the articles quoted in this proof the normalized local time is denoted by $L_{t}$ instead.) Let $\beta$ be the probability measure on $\mathrm{R}$ having the density function (also denoted by $\beta$ )

$$
\beta(b):=5 \sqrt{2} \mathbf{1}_{(-1 / 2,1 / 2)}(b)\left(\frac{1}{2}-|b|\right)^{3 / 2}, \quad b \in \mathrm{R} .
$$

Let $G$ denote a weak neighborhood of the law $\beta$. According to [12], Lemma 2.12 and [11], Section 4,

$$
\liminf _{t \uparrow \infty} \frac{1}{t} \log \Pi_{0,0}\left(t^{-1} L_{t} \in G, \tau_{1}>t\right) \geq-I(\beta),
$$

where

$$
I(\beta):=\frac{1}{8} \int d b \frac{\left[\beta^{\prime}(b)\right]^{2}}{\beta(b)}
$$


is a positive constant. Hence, there is a (positive) constant $c_{0}$ such that

$$
\Pi_{0,0}\left(\tau_{1}>t\right) \geq \exp \left(-c_{0} t\right) \text { for all large } t .
$$

On the other hand, for $\delta>0$, let $V(t, \delta)$ denote the event that for each $a$ and $b$ in R with $|a-b|<2 \delta$, we have $\left|L_{t}(a)-L_{t}(b)\right| \leq t$. Then by [12], Theorem 3.5,

$$
\lim _{\delta \downarrow 0} \limsup _{t \uparrow \infty} \frac{1}{t} \log \Pi_{0,0}\left(V(t, \delta)^{\mathrm{c}}\right)=-\infty
$$

In particular,

$$
\Pi_{0,0}\left(V(t, \delta)^{\mathrm{c}}\right) \leq \exp \left(-2 c_{0} t\right) \text { for all large } t \text { and small } \delta>0 .
$$

Using (123), there exists a constant, again denoted by $c_{0}$, such that

$$
\Pi_{0,0}\left(V(t, \delta) \cap\left\{\tau_{1}>t\right\}\right) \geq \Pi_{0,0}\left(\tau_{1}>t\right)-\Pi_{0,0}\left(V(t, \delta)^{\mathrm{c}}\right) \geq \exp \left(-c_{0} t\right)
$$

for all sufficiently large $t$ and small $\delta>0$. Fix such a $\delta$. Now on the event $V(t, \delta) \cap\left\{\tau_{1}>t\right\}$ we have $L_{t}(b) \leq 2 t / \delta$ since $L_{t}$ vanishes outside of $(-1,1)$ and by the definition of $V(t, \delta)$. Thus besides $c_{0}$ there is a constant $c_{1}=2 / \delta$ such that

$$
\Pi_{0,0}\left(\sup _{b} L_{t}(b) \leq c_{1} t, \tau_{1}>t\right) \geq \exp \left(-c_{0} t\right),
$$

for all $t>T$, say. By monotonicity, we may assume that $c_{1}=c_{0}$. Replacing $t$ by $t / \varepsilon^{2}$ for $t \geq T$ and $0<\varepsilon \leq 1$, and passing from $W_{r / \varepsilon^{2}}$ to $\varepsilon^{-1} W_{r}$ by selfsimilarity, changes $\tau_{1}>t / \varepsilon^{2}$ to $\tau_{\varepsilon}>t$, and $L_{t / \varepsilon^{2}}(b)$ to $\varepsilon^{-1} L_{t}(\varepsilon b)$. Therefore,

$$
\Pi_{0,0}\left(\varepsilon^{-1} \sup _{b} L_{t}(b) \leq \frac{c_{0} t}{\varepsilon^{2}}, \tau_{\varepsilon}>t\right) \geq \exp \left[-\frac{c_{0} t}{\varepsilon^{2}}\right],
$$

completing the proof.

Proof of Proposition 16. By monotonicity in $t$, it suffices to show the identity in (113) for each fixed $t \geq T$, with $T$ from Lemma 18, for almost all $\psi$. For this purpose, fix $t \geq T$ from now on. Since

$$
P_{0, \delta_{0}}\left(X_{t} \neq 0\right) \geq \widetilde{P}_{0, \delta_{0}}\left(\tilde{X}_{t}\left(A_{n}\right)>0\right), \quad n \geq 1,
$$

with

$$
A_{n}:=\left\{w \in \mathbf{C}: \sup _{b} L_{t}(b) \leq c_{0} t n, \tau_{1 / n}>t\right\}
$$

where $c_{0}$ is from Lemma 18 , it is enough to demonstrate that for almost all $\psi$,

$$
\liminf _{n \uparrow \infty} \widetilde{P}_{0, \delta_{0}}\left(\widetilde{X}_{t}\left(A_{n}\right)>0\right)=1 .
$$

To obtain this, fix $n \geq 1$ for the moment, and use the elementary inequality

$$
\widetilde{P}_{0, \delta_{0}}\left(\widetilde{X}_{t}\left(A_{n}\right)>0\right) \geq \frac{\left[\widetilde{P}_{0, \delta_{0}} \widetilde{X}_{t}\left(A_{n}\right)\right]^{2}}{\widetilde{P}_{0, \delta_{0}}\left[\widetilde{X}_{t}\left(A_{n}\right)\right]^{2}} .
$$


By the expectation formula (31),

$$
\widetilde{P}_{0, \delta_{0}} \widetilde{X}_{t}\left(A_{n}\right)=\widetilde{\Pi}_{0,0}\left(\widetilde{W}_{t} \in A_{n}\right) .
$$

On the other hand, by the covariance formula (32),

$$
\tilde{\operatorname{Var}}_{0, \delta_{0}} \tilde{X}_{t}\left(A_{n}\right) \leq 2 \widetilde{\Pi}_{0,0} \int_{0}^{t} L_{[W, \psi]}(d r) \widetilde{\Pi}_{r, \widetilde{W}_{r}}\left(\widetilde{W}_{t} \in A_{n}\right)=2\left\langle\psi, f_{n}\right\rangle,
$$

where

$$
f_{n}(b):=\widetilde{\Pi}_{0,0} \int_{0}^{t} d_{r} L_{r}(b) \widetilde{\Pi}_{r, \widetilde{W}_{r}}\left(\widetilde{W}_{t} \in A_{n}\right), \quad b \in \mathrm{R}, n \geq 1,
$$

(depending also on $t$ ). Assume for the moment that

$$
f_{n} \in L^{\infty}(\mathrm{R}) \text { and } 0<\left\|f_{n}\right\|_{\infty} \leq c_{0} \operatorname{tn} \widetilde{\Pi}_{0,0}\left(\tilde{W}_{t} \in A_{n}\right), \quad n \geq 1,
$$

with $c_{0}$ from Lemma 18. Then (133) implies

$$
\begin{aligned}
& \tilde{\operatorname{Var}}_{0,0} \tilde{X}_{t}\left(A_{n}\right) \\
& \quad \leq \frac{2\left\langle\psi, f_{n}\right\rangle}{\left\|f_{n}\right\|_{\infty} \exp \left[-2 c_{0} t n^{2}\right]} c_{0} t n \widetilde{\Pi}_{0,0}\left(\widetilde{W}_{t} \in A_{n}\right) \exp \left[-2 c_{0} t n^{2}\right],
\end{aligned}
$$

for all $n \geq 1$. Note that $f_{n}$ vanishes outside the interval $(-1 / n, 1 / n)$, by the definition of $A_{n}$. Set

$$
\mathscr{Q}:=\bigcup_{n \geq 1}\left\{b \in \mathrm{R}:\left|f_{n}(b)\right|>\left\|f_{n}\right\|_{\infty}\right\} .
$$

Fix now a realization $\psi$ of the catalyst such that $\psi(\mathscr{P})=0$ [recall (135) and (112)] and such that the identity in (111) is true for $K=2 c_{0} t$. Since

$$
\left\langle\psi, f_{n}\right\rangle=\int_{(-1 / n, 1 / n) \backslash \mathscr{g}} \psi(d b) f_{n}(b) \leq\left\|f_{n}\right\|_{\infty} \psi((-1 / n, 1 / n)),
$$

for a given $\delta>0$, we have

$$
\frac{2\left\langle\psi, f_{n}\right\rangle}{\left\|f_{n}\right\|_{\infty} \exp \left[-2 c_{0} t n^{2}\right]} \leq \delta
$$

for all $n$ sufficiently large, by the limit statement in (111). Additionally, we may choose $n$ so large that

$$
c_{0} t n \exp \left[-c_{0} t n^{2}\right] \leq \delta .
$$

Then, using Lemma 18 for the remaining term $\exp \left[-c_{0} t n^{2}\right]$, for these $n$, from (136) we get

$$
\widetilde{\operatorname{Var}_{0,0}} \tilde{X}_{t}\left(A_{n}\right) \leq \delta^{2}\left[\widetilde{\Pi}_{0,0}\left(\widetilde{W}_{t} \in A_{n}\right)\right]^{2} .
$$

Therefore, by (132),

$$
\widetilde{P}_{0, \delta_{0}}\left[\widetilde{X}_{t}\left(A_{n}\right)\right]^{2} \leq\left[\widetilde{\Pi}_{0,0}\left(\widetilde{W}_{t} \in A_{n}\right)\right]^{2}\left(1+\delta^{2}\right) .
$$


Hence, from (131) we obtain

$$
\liminf _{n \uparrow \infty} \widetilde{P}_{0, \delta_{0}}\left(\widetilde{X}_{t}\left(A_{n}\right)>0\right) \geq \frac{1}{1+\delta^{2}}, \quad \delta>0,
$$

which then yields (130).

It remains to verify (135) for $n \geq 1$ fixed. Let us first do this by a formal calculation using $\delta$-functions. In fact, by the definition (134) of $f_{n}$, formally

$$
\begin{aligned}
f_{n}(b) & =\widetilde{\Pi}_{0,0} \int_{0}^{t} d r \delta_{b}\left(W_{r}\right) \widetilde{\Pi}_{r, \widetilde{W}_{r}} \mathbf{1}_{A_{n}}\left(\widetilde{W}_{t}\right) \\
& =\int_{0}^{t} d r \widetilde{\Pi}_{0,0} \widetilde{\Pi}_{r, \widetilde{W}_{r}} \delta_{b}\left(W_{r}\right) \mathbf{1}_{A_{n}}\left(\widetilde{W}_{t}\right) \\
& =\widetilde{\Pi}_{0,0} L_{t}(b) \mathbf{1}_{A_{n}}\left(\widetilde{W}_{t}\right) .
\end{aligned}
$$

But on $A_{n}$ [defined in (129)], $L_{t}(b) \leq c_{0} t n$, and (135) follows (the positivity of $\left\|f_{n}\right\|_{\infty}$ is obvious).

To make the previous argument rigorous, we consider the functional

$$
F(g):=\int d b g(b) f_{n}(b), \quad g \in L^{\mathbf{1}}(\mathrm{R}) .
$$

We want to show that $g \mapsto F(g)$ is a bounded linear functional on $L^{\mathbf{1}}(\mathrm{R})$ with operator norm

$$
\|F\| \leq c_{0} \operatorname{tn} \widetilde{\Pi}_{0,0}\left(\widetilde{W}_{t} \in A_{n}\right) .
$$

Then, by the Riesz representation theorem, (135) is true. Now from the definition (134) of $f_{n}$,

$$
\begin{aligned}
|F(g)| & \leq \int d b|g(b)| \widetilde{\Pi}_{0,0} \int_{0}^{t} d_{r} L_{r}(b) \widetilde{\Pi}_{r, \widetilde{W}_{r}} \mathbf{1}_{A_{n}}\left(\widetilde{W}_{t}\right) \\
& =\widetilde{\Pi}_{0,0} \int_{0}^{t} d r\left|g\left(W_{r}\right)\right| \widetilde{\Pi}_{r, \widetilde{W}_{r}} \mathbf{1}_{A_{n}}\left(\widetilde{W}_{t}\right) \\
& =\int_{0}^{t} d r \widetilde{\Pi}_{0,0} \widetilde{\Pi}_{r, \widetilde{W}_{r}}\left|g\left(W_{r}\right)\right| \mathbf{1}_{A_{n}}\left(\widetilde{W}_{t}\right) \\
& =\widetilde{\Pi}_{0,0} \int_{0}^{t} d r\left|g\left(W_{r}\right)\right| \mathbf{1}_{A_{n}}\left(\widetilde{W}_{t}\right) .
\end{aligned}
$$

But on $A_{n}$,

$$
\int_{0}^{t} d r\left|g\left(W_{r}\right)\right|=\int d b L_{t}(b)|g(b)| \leq c_{0} t n\|g\|_{1} .
$$

Hence, from (147),

$$
|F(g)| \leq c_{0} t n\|g\|_{1} \widetilde{\Pi}_{0,0}\left(\widetilde{W}_{t} \in A_{n}\right),
$$

and the claim (146) follows. This completes the proof. 
5. Proof of the main result. The proof of Theorem 6 concerning finite time extinction of the one-dimensional SBM $X^{\Gamma}$ with a stable catalyst proceeds in several steps. Since here we start with an initial measure $\mu$ of compact support, and $X^{\Gamma}$ has the compact support property [9], by some stopping argument we will pass to a periodic catalyst $\Gamma^{\mathrm{K}}$. Moreover, because the survival probability is monotone in the catalyst, we will switch to a smaller catalyst, as already explained in Section 1.1. Altogether we will reduce to the case of a point catalyst $\underline{\Gamma}$ with dense locations as dealt with in Section 4.2.

5.1. Passing to a periodic catalyst. Recall that the historical catalytic SBM $\tilde{X}^{\Gamma}$ exists for $\mathbb{P}$-almost all $\Gamma$. Fix an initial measure $\mu \in \mathscr{M}_{f}$ with compact support. We want to show that

$$
\lim _{t \uparrow \infty} \widetilde{P}_{0, \mu}^{\Gamma}\left(\widetilde{X}_{t}^{\Gamma} \neq 0\right)=0, \quad \mathbb{P} \text {-a.s. }
$$

For $\mathrm{K} \geq 1$, set

$$
\mathbf{C}_{\mathrm{K}}:=\left\{w \in \mathbf{C}:\left|w_{s}\right|<\mathrm{K}, \forall s \geq 0\right\}
$$

According to the compact support property established in [9],

$$
\liminf _{\mathrm{K} \uparrow \infty} \widetilde{P}_{t \geq 0}^{\Gamma}\left(\operatorname{supp} \widetilde{X}_{t}^{\Gamma} \subseteq \mathbf{C}_{\mathrm{K}}\right)=1 \quad \text { for each } t \geq 0, \quad \mathbb{P} \text {-a.s. }
$$

For the further proof, fix such a sample $\Gamma$. By (152), instead of (150) it suffices to show that

$$
\lim _{t \uparrow \infty} \widetilde{P}_{0, \mu}^{\Gamma}\left(\widetilde{X}_{t}^{\Gamma} \neq 0 \text { and supp } \widetilde{X}_{t}^{\Gamma} \subseteq \mathbf{C}_{\mathrm{K}}\right)=0 \text { for all large } \mathrm{K} .
$$

However, under this restriction to historical paths $w$ living in $\mathbf{C}_{\mathrm{K}}$, we may change the catalyst outside of $[-\mathrm{K}, \mathrm{K}]$ without affecting the latter probability. This will be formalized in the following considerations.

Fix $K \geq 1$ such that the initial measure $\mu$ is supported by $(-K, K)$, and that there is no catalyst's atom at the boundary $\{-\mathrm{K}, \mathrm{K}\}$ of the interval $(-\mathrm{K}, \mathrm{K})$, that is, $\Gamma(\{-\mathrm{K}, \mathrm{K}\})=0$ (this holds with $\mathbb{P}$-probability 1$)$. Consider the hitting time $\tau^{\mathrm{K}}$ of $\{-\mathrm{K}, \mathrm{K}\}$ and the stopped historical SBM $t \mapsto \tilde{X}_{t \wedge \tau^{\mathrm{K}}}^{\Gamma}$, as in (34).

We need also another stopped historical SBM: $t \mapsto \widetilde{X}_{t \wedge \tau^{\mathrm{K}}}^{\Gamma^{\mathrm{K}}}$. Here $\Gamma^{\mathrm{K}}$ denotes the periodic extension of the restriction $\Gamma((\cdot) \cap(-\mathrm{K}, \mathrm{K}])$ of $\Gamma$ to $(-\mathrm{K}, \mathrm{K}]$ to all of $\mathrm{R}$ (for the fixed $\Gamma$ ).

LEMMA 19 (Passing to a periodic catalyst). Fix $\mathrm{K} \geq 1$ such that the initial measure $\mu$ is supported by $(-\mathrm{K}, \mathrm{K})$. Given $\Gamma$,

$$
\widetilde{P}_{0, \mu}^{\Gamma}\left(\widetilde{X}_{t \wedge \tau^{K}}^{\Gamma} \in \cdot\right)=\widetilde{P}_{0, \mu}^{\Gamma^{K}}\left(\widetilde{X}_{t \wedge \tau^{\kappa}}^{\Gamma^{K}} \in \cdot\right), \quad t \geq 0 .
$$


Proof. Apply the log-Laplace representation (35) and (36) with $K=L_{[W, \Gamma]}$ and $K=L_{\left[W, \Gamma^{\mathrm{K}}\right]}$, respectively, and employ the identity

$$
\mathbf{1}_{\left\{s \leq t \wedge \tau^{K}\right\}} L_{[W, \Gamma]}(d s)=\mathbf{1}_{\left\{s \leq t \wedge \tau^{\mathrm{K}\}}\right.} L_{\left[W, \Gamma^{\mathrm{K}}\right]}(d s) .
$$

This immediately gives the claim.

Now we use the latter lemma to pass in (153) from $\Gamma$ to the periodic $\Gamma^{\mathrm{K}}$. In fact, supp $\widetilde{X}_{t}^{\Gamma} \subseteq \mathbf{C}_{\mathrm{K}}$ implies that for $w \in \operatorname{supp} \widetilde{X}_{t}^{\Gamma}$ we have $\tau_{\mathrm{K}}>t$, hence $\tau_{\mathrm{K}} \wedge t=$ $t$, consequently in (153) we can replace $\tilde{X}_{t}^{\Gamma}$ by $\tilde{X}_{t \wedge \tau_{K}}^{\Gamma}$. But then by Lemma 19 , we may pass even to $\widetilde{X}_{t \wedge \tau_{\mathrm{K}}}^{\Gamma^{\mathrm{K}}}$ and going back to $\tilde{X}_{t}^{\Gamma^{\mathrm{K}}}$ by the same reasoning, we see that instead of (153) it suffices to show

$$
\lim _{t \rightarrow \infty} \widetilde{P}_{0, \mu}^{\Gamma^{K}}\left(\tilde{X}_{t}^{\Gamma^{K}} \neq 0\right)=0
$$

for each fixed $\mathrm{K} \geq 1$ such that $\mu$ is supported by $(-\mathrm{K}, \mathrm{K})$ and that $\Gamma(\{-\mathrm{K}, \mathrm{K}\})=0$. In other words, we want to show finite time extinction of the historical catalytic SBM $\widetilde{X}^{\Gamma^{\mathrm{K}}}$ with fixed periodic catalyst $\Gamma^{\mathrm{K}}$, which will be done in the next subsection.

5.2. Completion of the proof of the main theorem. In order to apply later on the result of Section 4.2, we further use the fact that the collision local times $L_{[W, \psi]}$ are nondecreasing in $\psi \in \mathscr{M}_{p}$. That is, $\psi_{1} \leq \psi_{2}$ implies $L_{\left[W, \psi_{1}\right]} \leq L_{\left[W, \psi_{2}\right]}$. Therefore the corresponding solutions $v^{\psi}$ of the log-Laplace equation (29) with $K$ replaced by $L_{[W, \psi]}$ are nonincreasing: $v^{\psi^{1}} \geq v^{\psi^{2}}$. But this yields that the extinction probability is nondecreasing in $\psi$ [recall (4)].

LEMMA 20 (Comparison of extinction probabilities). Let $\psi_{1}$ and $\psi_{2}$ belong to $\mathscr{M}_{p}$ and satisfy $\psi_{1} \leq \psi_{2}$. Then

$$
\widetilde{P}_{0, \mu}^{\psi_{1}}\left(\tilde{X}_{t}=0\right) \leq \widetilde{P}_{0, \mu}^{\psi_{2}}\left(\tilde{X}_{t}=0\right)
$$

for all $\mu \in \mathscr{M}_{f}$ and $t \geq 0$.

Hence, for our purpose of verifying (156), we may replace the periodic catalyst $\Gamma^{\mathrm{K}}$ by a smaller measure.

To this end, as already mentioned in Section 1.1, we first drop all the "big" point catalysts: For the moment, fix $N \geq 0$ (independent of $\varepsilon$ ) and remove all those atoms $\alpha_{i} \delta_{b_{i}}$ of $\Gamma^{\mathrm{K}}$ [or $\Gamma$, recall the representation (22)] with action weight $\alpha_{i} \geq 2^{-N+1}$. Next, for each $n \geq N$, we replace the action weights $\alpha_{i} \in\left[2^{-n}, 2^{-n+1}\right)$ by $2^{-n}$. Note that with respect to $\mathbb{P}$, the positions $b_{i} \in(-\mathrm{K}, \mathrm{K}]$ of the related atoms are distributed as a Poisson point process with intensity measure $c_{\gamma} 2^{\gamma n} \mathbf{1}_{(-\mathrm{K}, \mathrm{K}]}(b) \ell(d b)$. Here the constant $c_{\gamma}$ is given by

$$
c_{\gamma}:=\gamma^{-1}\left(1-2^{-\gamma}\right)\left(\int_{0}^{\infty} d r r^{-1-\gamma}\left(1-e^{-r}\right)\right)^{-1}
$$


(see, e.g., [4]). Let $\underline{\pi}_{n}$ denote the periodic extension of this Poisson point process, extension from $(-K, K]$ to all of $R$.

What remains for the reduction to Section 4.2 is to show that $\mathbb{P}$-a.s. in $\underline{\pi}_{n}$ neighboring catalysts have a distance of at most $\Delta_{n}=e^{-\beta n}$, for all $n \geq N$, for $N \geq 0$ appropriately chosen. For this purpose, we fix

$$
\beta \in(0, \gamma \log 2) .
$$

By Borel-Cantelli, it suffices to show that the quantities

$$
\mathbb{P}\left(\exists \text { two neighboring points in } \underline{\pi}_{n} \text { with a distance larger than } e^{-\beta n}\right)
$$

are summable in $n \geq 1$. But each of these probabilities is bounded from above by

$$
\mathbb{P}\left(\max _{1 \leq i \leq J_{n}+1} \xi_{i}>e^{-\beta n}\right)
$$

where $J_{n}$ is the Poissonian number of points in $(-\mathrm{K}, \mathrm{K}]$ with expectation $a_{n}:=$ $2 \mathrm{~K} c_{\gamma} 2^{\gamma n}$, and the $\xi_{1}, \xi_{2}, \ldots$ are i.i.d. exponentials with parameter $a_{n}$. Now the $J_{n}$ satisfy a standard large deviation principle as $n \uparrow \infty$, hence,

$$
\mathbb{P}\left(J_{n}+1>2 a_{n}\right) \leq \exp \left[-c 2^{\gamma n}\right]
$$

for all sufficiently large $n$. Since the right-hand side is summable in $n$, in the probability expression (161) we may additionally restrict to $J_{n}+1 \leq 2 a_{n}$. Consequently, instead of (161) we look at

$$
\mathbb{P}\left(\max _{1 \leq i \leq 2 a_{n}} \xi_{i}>e^{-\beta n}\right) \text {. }
$$

By scaling, we may switch to

$$
\mathscr{P}\left(\max _{1 \leq i \leq 2 a_{n}} \xi_{i}^{\prime}>a_{n} e^{-\beta n}\right),
$$

where the $\xi_{i}^{\prime}$ are now i.i.d. standard exponentials (under the law denoted by $\mathscr{P})$.

Next we use the fact that all $x \geq 0$ and $m \geq 2$,

$$
\left|\mathscr{P}\left(\max _{1 \leq i \leq m} \xi_{i}^{\prime}-\log m>x\right)-\left(1-\exp \left[-e^{-x}\right]\right)\right| \leq 2 e^{-2 x}
$$

(see Example 2.10.1 in [23]; take $q=\frac{1}{2}$ there). Now, for all $n$ sufficiently large, $m=\left[2 a_{n}\right]$ and

(166) $x=a_{n} e^{-\beta n}-\log \left(2 a_{n}\right)=c \exp (n(\gamma \log 2-\beta))-\log \left(4 \mathrm{~K} c_{\gamma}\right)-n \gamma \log 2$

satisfy these conditions [recall (159)]. Thus, for (164) we get the bound

$$
1-\exp \left[-e^{-x}\right]+2 e^{-2 x} \leq 3 e^{-x},
$$

which for $x$ from (166) is summable in $n$.

This completes the proof of Theorem 6 . 
6. The lattice model. Now consider the model with random catalysts on the lattice $Z^{d}$. Recall that $\varrho=\left\{\varrho_{b}\right\}_{b \in Z^{d}}$, the catalysts, are i.i.d. random variables which are uniformly distributed on $[0,1]$. Instead of Brownian motions, the motion process is now given by a continuous time simple random walk on $Z^{d}$, which moves to a neighboring site at rate 1 . In other words, the times between jumps are i.i.d. exponential waiting times with mean 1.

We use symbols analogous to the ones in earlier sections. In particular, $\mathbb{P}$ denotes the law of the catalyst, $W=\left[W, \Pi_{s, a}, s \geq 0, a \in Z^{d}\right]$ the simple random walk in $Z^{d}$ on canonical Skorohod path space $\mathbf{D}=\mathscr{D}\left[R_{+}, Z^{d}\right]$ of cadlag functions, and

$$
\tilde{X}^{\varrho}=\left[\tilde{X}^{\varrho}, \widetilde{P}_{s, \mu}^{\varrho}, s \geq 0, \mu \in \mathscr{M}_{f}\left[\mathbf{D}^{s}\right]\right]
$$

the historical simple super-random walk on $Z^{d}$ (also called simple interacting Feller's branching diffusion) with catalyst $\varrho$. Note that Proposition 7, Theorem 10, Lemma 11 and Proposition 12 remain valid (with the obvious changes).

For simplicity, we now assume that $X_{0}=\delta_{0}$. Our aim is to show the finite time extinction property for $\tilde{X}^{\varrho}$, for $\mathbb{P}$-a.a. $\varrho$. In this case, the bad historical paths are those which spend a large amount of time at sites $b \in Z^{d}$ where $\varrho_{b}$ is small. We will choose time $T_{N}$ so that, with high probability, most of the mass is dead by this time [in the sense of (42)]. This is the hardest part of the argument. Bounding the mass after this uses similar but easier ideas.

We also need the following crude estimate on the distance traveled by the simple random walk $W$ in time $t$. Let $J_{t}$ denote the number of jumps taken by $W$ by time $t$. Since $J_{t}$ is Poisson with parameter $t$, for $k \geq 0$ we have

$$
\begin{aligned}
\Pi_{0,0}\left(\sup _{0 \leq s \leq t}\left|W_{s}\right| \geq k\right) & \leq \Pi_{0,0}\left(J_{t} \geq k\right)=e^{-1} \sum_{i=k}^{\infty} \frac{t^{i}}{i !} \\
& \leq e^{-1} \frac{t^{k}}{k !} \sum_{i=0}^{\infty} \frac{t^{i}}{i !}=\frac{t^{k}}{k !} \leq\left(\frac{t e}{k}\right)^{k}(2 \pi k)^{-1 / 2},
\end{aligned}
$$

the latter by Stirling's approximation.

For $n \geq 0$, let $D_{n}$ denote the cube

$$
D_{n}=\left\{\left(b_{1}, \ldots, b_{d}\right) \in Z^{d}: \max \left(\left|b_{1}\right|, \ldots,\left|b_{d}\right|\right) \leq 2^{n}\right\}
$$

in $Z^{d}$ having $\left(2^{n+1}+1\right)^{d}$ sites. For a given path $w \in \mathbf{D}$, let $\tau_{n}=\tau_{n}(w)$ denote the first time $t \geq 0$ that $w_{t}$ does not belong to $D_{n}$. We intend to use Dynkin's special Markov property to start the stopped historical super-random walk $\left\{\widetilde{X}_{\tau_{n}}^{o}: n \geq 0\right\}$ afresh at the times $\tau_{n}$.

We define in this proof that the quantities $M_{n}^{\varepsilon}, \lambda_{n}^{\varepsilon}, \xi_{n}^{\varepsilon}, \delta_{n}^{\varepsilon}, T_{n}^{\varepsilon}$ and $E_{n}^{\varepsilon}$ entering in Hypotheses 8 and 9 to be independent of $\varepsilon$, and therefore we omit the index $\varepsilon$. On the other hand, for $0<\varepsilon<1$, we will choose $N=N(\varepsilon) \geq 1$ later, such that $\lim _{\varepsilon \downarrow 0} N(\varepsilon)=\infty$. To be more specific, $N$ must be so large that all of the statements involving the phrase "for $N$ sufficiently large" are 
satisfied. Set

$$
\delta_{N-1}:=2^{-N / 4}, \quad T_{N}:=\frac{2^{N}}{6} \wedge \tau_{N}
$$

and for $n \geq N=N(\varepsilon)$, let

$$
\begin{array}{ll}
M_{n}:=2^{-n(d+3)}, & \lambda_{n}:=2^{-2^{n}}, \quad \delta_{n}:=2^{-n}, \\
\xi_{n}:=2^{-n(d+2)}, & T_{n+1}:=\left(T_{n}+2^{-n}\right) \wedge \tau_{n+1} .
\end{array}
$$

Note that $\delta_{N-1}$ and $T_{N}$ implicitly depend on $\varepsilon$ via $N(\varepsilon)$. One can easily show that $T_{n+1}>T_{n}, \Pi_{0,0}$-a.s. Clearly (b1) and (b2) of Hypothesis 8 are satisfied.

We need the following large deviations lemma on the simple random walk $W$. We say that a nonempty subset $S \subset Z^{d}$ is connected if any two elements $a, b \in S$ are connected by a chain $a=z_{0}, \ldots, z_{k}=b$ of elements of $S$, such that for $1 \leq i \leq k$ the points $z_{i-1}, z_{i}$ are nearest neighbors. That is, they are distance 1 apart.

LEMMA 21 (Large deviations). Fix $m \geq 1$. Suppose that $S \subset Z^{d}$ has the property that no connected subset of $S$ has cardinality larger than $m$. Then there exist constants $\alpha_{0}, c_{0}>0$ (depending on $m$ ) such that for all $t \geq 1$,

$$
\sup _{a \in Z^{d}} \Pi_{0, a}\left(\int_{0}^{t} d s \mathbf{1}_{\left\{W_{s} \in S^{c}\right\}} \leq \alpha_{0} t\right) \leq c_{0}^{-1} e^{-c_{0} t} .
$$

PROOF. By monotonicity in $m$, we may enlarge $m$ if necessary, so we can assume that $m$ is even. To each $a \in Z^{d}$, there exists a chain consisting of points $a=z_{0}, \ldots, z_{m}$ such that $z_{i-1}, z_{i}$ are nearest neighbors for $1 \leq i \leq m$, and $z_{m}=z_{m}(a) \in S^{\mathrm{c}}$. In fact, if $a \in S$, this follows from our assumption on $S$. Otherwise, let $b$ be one of the nearest neighbors of $a$, and set $z_{2 k}:=a$, $z_{2 k+1}:=b$ for $0 \leq k<m / 2$.

Suppose that $W_{0}=a$. Let $\eta_{a}$ denote the first time $t$ that $W_{t}=z_{m}(a)$. (If there is no such time, set $\eta_{a}=\infty$.) Let $F=F(a)$ denote the event that $\eta_{a}<1 / 2$ and that $W_{s}=z_{m}(a)$ for $\eta_{a} \leq s \leq 1$. By the properties of our continuous-time simple random walk, using the constructed chain, there exists $\alpha_{0}>0$ such that for all $a \in Z^{d}$,

$$
\Pi_{0, a}(F(a)) \geq 8 \alpha_{0} .
$$

Let $F_{i}:=\theta_{i} F, i \geq 0$, where $\theta_{s}$ is the time-shift operator on paths. By the Markov property and (175), given $a \in Z^{d}$ there exists a sequence of independent events $\bar{F}_{i}$ such that $\bar{F}_{i} \subset F_{i}$ and $\Pi_{0, a}\left(\bar{F}_{i}\right)=8 \alpha_{0}$, for each $i$. Set

$$
G_{k}:=\sum_{i=0}^{k-1} \mathbf{1}_{\bar{F}_{i}} .
$$


By Chernoff's large deviations theorem (see [2], Theorem 9.3), there exist a constant $c_{0}>0$ such that for all $a \in Z^{d}$, we have

$$
\Pi_{0, a}\left(\frac{G_{k}}{k} \leq 4 \alpha_{0}\right) \leq c_{0}^{-1} \exp \left(-c_{0} k\right), \quad k \geq 0 .
$$

Note that

$$
\int_{i}^{i+1} d s \mathbf{1}_{\left\{W_{s} \in S^{\mathrm{c}}\right\}} \geq 1 / 2 \text { on } F_{i} .
$$

Indeed, if $F_{i}$ occurs, then $W_{s} \in S^{\mathrm{c}}$ for $s \in(i+1 / 2, i+1)$. Suppose that $G_{k} / k \geq 4 \alpha_{0}$. Then there are at least $4 \alpha_{0} k$ indices $i \leq k-1$ such that $\bar{F}_{i}$ occurs, and hence $F_{i}$ occurs. In that case, by (178),

$$
\int_{0}^{k} d s \mathbf{1}_{\left\{W_{s} \in S^{\mathrm{c}}\right\}} \geq 2 \alpha_{0} k \text {. }
$$

Hence, for $a \in Z^{d}$ and $k \geq 0$,

$$
\Pi_{0, a}\left(\frac{G_{k}}{k}>4 \alpha_{0}\right) \leq \Pi_{0, a}\left(\int_{0}^{k} d s \mathbf{1}_{\left\{W_{s} \in S^{c}\right\}} \geq 2 \alpha_{0} k\right) .
$$

Interpolating, we have that for all $a \in Z^{d}$ and $t \geq 1$,

$$
\begin{aligned}
\Pi_{0, a}\left(\int_{0}^{t} d s \mathbf{1}_{\left\{W_{s} \in S^{\mathrm{c}}\right\}}<\alpha_{0} t\right) & \leq \Pi_{0, a}\left(\int_{0}^{[t]} d s \mathbf{1}_{\left\{W_{s} \in S^{\mathrm{c}}\right\}}<\alpha_{0}([t]+1)\right) \\
& \leq c_{0}^{-1} e^{-c_{0} t},
\end{aligned}
$$

completing the proof of Lemma 21.

Next we want to show that the event that the catalyst is small on large connected sets has small probability; see (191) below. For $m \geq 1,0<\varepsilon<1$, $n \geq N=N(\varepsilon) \geq 1$, and $0 \leq \zeta \leq 1$, let $A(m, n, \zeta)$ denote the (catalyst) event that there is no connected subset $S \subset D_{n}$ with cardinality $m$, on which all of the catalysts satisfy $\varrho_{b} \leq \zeta$. Note that there is a finite number $c(m, d)$ of connected sets of cardinality $m$, which contain a given point. Then we have

$$
\begin{aligned}
\mathbb{P}\left(A^{\mathrm{c}}(m, n, \zeta)\right) & \leq\left(2^{n+1}+1\right)^{d} c(m, d)\left(\mathbb{P}\left(\varrho_{b} \leq \zeta\right)\right)^{m} \\
& =\left(2^{n+1}+1\right)^{d} c(m, d) \zeta^{m} .
\end{aligned}
$$

In particular, if

$$
\zeta=\zeta_{n}=2^{-(n-1)(d+1)},
$$

then

$$
\mathbb{P}\left(A^{\mathrm{c}}\left(1, n, \zeta_{n}\right)\right) \leq c 2^{n d} 2^{-(n-1)(d+1)}=c 2^{-n} .
$$

For $m=1$, all catalysts in $D_{n}$ are greater than $\zeta$ on $A(1, n, \zeta)$. Put

$$
A_{1}(n):=\bigcap_{k=n}^{\infty} A\left(1, k, \zeta_{k}\right)
$$


and note that

$$
\mathbb{P}\left(A_{1}^{\mathrm{c}}(n)\right) \leq c_{1} 2^{-n}
$$

for some constant $c_{1}$.

From now on, let

$$
m=2(d+1)
$$

and take

$$
\bar{\zeta}=\bar{\zeta}_{n}=2^{-n / 2}
$$

Then, by (181), we have,

$$
\mathbb{P}\left(A^{\mathrm{c}}\left(m, n, \bar{\zeta}_{n}\right)\right) \leq c 2^{-n}
$$

Let

$$
A_{2}(n):=\bigcap_{k=n}^{\infty} A\left(m, k, \bar{\zeta}_{k}\right)
$$

and note that

$$
\mathbb{P}\left(A_{2}^{\mathrm{c}}(n)\right) \leq c_{2} 2^{-n}
$$

for some constant $c_{2}$. Fix $\bar{\varepsilon}>0$. Using (185) and (190), we choose $\bar{n}=\bar{n}(\bar{\varepsilon})$ so large that

$$
\mathbb{P}\left(A_{1}^{\mathrm{c}}(\bar{n}) \cup A_{2}^{\mathrm{c}}(\bar{n})\right) \leq\left(c_{1}+c_{2}\right) 2^{-\bar{n}}<\bar{\varepsilon} .
$$

We will apply our general Theorem 10 with $N$ chosen to satisfy $N=$ $N(\varepsilon, \bar{\varepsilon}) \geq \bar{n}$. We will conclude that for catalysts $\varrho$ in $A_{\mathbf{1}}(\bar{n}) \cap A_{\mathbf{2}}(\bar{n})$, finite time extinction occurs with $\widetilde{P}_{0, \mu}^{\varrho}$-probability 1 . Therefore, with $\mathbb{P}$-probability at least $1-\bar{\varepsilon}$, finite time extinction occurs. Since $\bar{\varepsilon}$ is arbitrary, our proof will then be finished.

From now on we assume that the catalyst $\varrho$ belongs to the set $A_{1}(\bar{n}) \cap A_{2}(\bar{n})$. In order to verify the starting condition (42) in Hypothesis 9, we extend the definition (41) of good historical paths in writing $E_{N-1}$ for the set of paths $w$ such that

$$
\int_{0}^{T_{N}} L_{[w, \varrho]}(d s) \geq \xi_{N-1}:=\frac{\alpha_{0} 2^{N / 2}}{6}
$$

with $\alpha_{0}$ from Lemma 21. Let $\bar{T}_{N}:=2^{N} / 6$. Recall that $T_{N}=\bar{T}_{N} \wedge \tau_{N}$, and note that $\xi_{N-1}=\alpha_{0} \bar{T}_{N} \bar{\zeta}_{N}$ with $\bar{\zeta}_{N}$ from (187). Then

$$
\begin{aligned}
\Pi_{0,0}\left(E_{N-1}^{\mathrm{c}}\right) \leq & \Pi_{0,0}\left(\int_{0}^{T_{N}} L_{[W, \varrho]}(d s) \leq \alpha_{0} \bar{T}_{N} \bar{\zeta}_{N}\right) \\
\leq & \Pi_{0,0}\left(\tau_{N} \leq \bar{T}_{N}\right) \\
& +\Pi_{0,0}\left(\int_{0}^{T_{N}} L_{[W, \varrho]}(d s) \leq \alpha_{0} \bar{T}_{N} \bar{\zeta}_{N}, \tau_{N}>\bar{T}_{N}\right) .
\end{aligned}
$$


Hence,

$$
\Pi_{0,0}\left(E_{N-1}^{\mathrm{c}}\right) \leq \Pi_{0,0}\left(\tau_{N} \leq \bar{T}_{N}\right)+\Pi_{0,0}\left(\int_{0}^{\bar{T}_{N}} L_{[W, \varrho]}(d s) \leq \alpha_{0} \bar{T}_{N} \bar{\zeta}_{N}\right)
$$

Below we will use (169) to handle the first term at the right-hand side. In order to deal with the second term, let $\bar{\varrho}=\bar{\varrho}(N)$ be obtained from $\varrho$ as follows. Put $\bar{\varrho}_{b}:=\varrho_{b}$ if $b \in D_{N}$. Otherwise, set $\bar{\varrho}_{b}:=1$. Let $S_{N}$ denote the collection of sites $b \in Z^{d}$ such that $\bar{\varrho} \leq \bar{\zeta}_{N}$ [recall notation (187)]. Note that by the definition of $A\left(m, N, \bar{\zeta}_{N}\right) \supseteq A_{2}(\bar{n})$, there is no connected subset of $S_{N}$ with cardinality $N$. Thus, by Lemma 21 ,

$$
\Pi_{0,0}\left(\int_{0}^{\bar{T}_{N}} d s \mathbf{1}_{\left\{W_{s} \in S^{c}\right\}} \leq \alpha_{0} \bar{T}_{N}\right) \leq c_{0}^{-1} \exp \left[-c_{0} \bar{T}_{N}\right]
$$

By the definition of $S_{N}$.

$$
\int_{0}^{\bar{T}_{N}} d s \mathbf{1}_{\left\{W_{s} \in S_{N}^{\mathrm{c}}\right\}}>\alpha_{0} \bar{T}_{N} \quad \text { implies } \int_{0}^{\bar{T}_{N}} L_{[w, \bar{\varrho}]}(d s)>\alpha_{0} \bar{T}_{N} \bar{\zeta}_{N},
$$

and so

$$
\begin{gathered}
\Pi_{0,0}\left(\int_{0}^{\bar{T}_{N}} L_{[W, \bar{\varrho}]}(d s) \leq \alpha_{0} \bar{T}_{N} \bar{\zeta}_{N}\right) \\
\quad \leq \Pi_{0,0}\left(\int_{0}^{\bar{T}_{N}} d s \mathbf{1}_{\left\{W_{s} \in S_{N}^{\mathrm{c}}\right\}} \leq \alpha_{0} \bar{T}_{N}\right) .
\end{gathered}
$$

Therefore,

$$
\Pi_{0,0}\left(\int_{0}^{\bar{T}_{N}} L_{[W, \bar{\varrho}]}(d s) \leq \alpha_{0} \bar{T}_{N} \bar{\zeta}_{N}\right) \leq c_{0}^{-1} \exp \left[-c_{0} \bar{T}_{N}\right] .
$$

Now (193) and the previous estimate combined with (169) gives

$$
\begin{aligned}
\Pi_{0,0}\left(E_{N-1}^{\mathrm{c}}\right) & \leq \Pi_{0,0}\left(\tau_{N} \leq \bar{T}_{N}\right)+c_{0}^{-1} \exp \left[-c_{0} \bar{T}_{N}\right] \\
& \leq\left(\frac{2^{N}(e / 6)}{2^{N}}\right)^{2^{N}}\left(2 \pi 2^{N}\right)^{-1 / 2}+c_{0}^{-1} \exp \left[-c_{0} \frac{2^{N}}{6}\right] \\
& \leq \exp \left[-2 c_{3} 2^{N}\right]
\end{aligned}
$$

for some constant $c_{3}$ (increasing $N$ if necessary). Therefore, by Markov's inequality, and the "stopped expectation" formula (37),

$$
\widetilde{P}_{0, \delta_{0}}^{\varrho}\left(\widetilde{X}_{T_{N}}^{\varrho}\left(E_{N-1}^{\mathrm{c}}\right)>\exp \left[-c_{3} 2^{N}\right]\right) \leq \exp \left[-c_{3} 2^{N}\right] .
$$

Next we consider $\tilde{X}_{T_{N}}^{\varrho}\left(E_{N-1}\right)$. We wish to show that in the case $K=L_{[W, \varrho]}$, condition (62) in Proposition 12 holds $\mathbb{P}$-a.s. By Fubini's theorem, it suffices to verify it $\Pi_{0,0} \times \mathbb{P}$-a.s. First note that with $\Pi_{0,0}$-probability 1 the range $\mathscr{R}(W)$ of the random walk $W$ is infinite. For each site $b \in \mathscr{R}(W)$, let $Y_{b}:=\sigma_{b} \varrho_{b}$, where $\sigma_{b}$ is the amount of time which $W$ spends at $b$ between the time of first 
arrival at $b$ and the first subsequent departure. Then the $Y_{b}$ are i.i.d. with positive $\Pi_{0,0} \times \mathbb{P}$-expectation. Therefore, by the strong law,

$$
\int_{0}^{\infty} L_{[W, \varrho]}(d s) \geq \sum_{b \in \mathscr{R}(W)} Y_{b}=\infty, \quad \Pi_{0,0} \times \mathbb{P} \text {-a.s. },
$$

giving (62).

By Proposition 12 with

$$
T_{0}=0, \quad T_{1}=T_{N-1}, \quad \xi=\xi_{N-1}=\frac{\alpha_{0}}{6} 2^{N / 2} \quad \text { and } \quad E=E_{N-1},
$$

we obtain

$$
\widetilde{P}_{0, \delta_{0}}^{\varrho}\left(\widetilde{X}_{T_{N}}^{\varrho}\left(E_{N-1}\right)>0\right) \leq \frac{1}{\xi_{N-1}}=\frac{6}{\alpha_{0}} 2^{-N / 2} .
$$

If $N$ is large enough, we have $M_{N} \geq \exp \left[-c_{3} 2^{N}\right]$. Hence, by (198) and (200),

$$
\begin{aligned}
\widetilde{P}_{0, \delta_{0}}^{\varrho}\left(\left\|\widetilde{X}_{T_{N}}^{\varrho}\right\|>M_{N}\right) \leq & \widetilde{P}_{0, \delta_{0}}^{\varrho}\left(\tilde{X}_{T_{N}}^{\varrho}\left(E_{N-1}^{\mathrm{c}}\right)>\exp \left[-c_{3} 2^{N}\right]\right) \\
& +\widetilde{P}_{0, \delta_{0}}^{\varrho}\left(\tilde{X}_{T_{N}}^{\varrho}\left(E_{N-1}\right)>0\right) \\
\leq & \exp \left[-c_{3} 2^{N}\right]+\frac{6}{\alpha_{0}} 2^{-N / 2} \leq 2^{-N / 4}=\delta_{N-1} .
\end{aligned}
$$

This implies the starting condition (42) in Hypothesis 9.

Now we consider the other time intervals $\left[T_{n}, T_{n+1}\right), n \geq N$. To deal with these times, we no longer consider clusters of sites where the catalyst is small, but consider just single sites. Recall that we are on the set $A_{1}(\bar{n})$ and that $n \geq N \geq \bar{n}$. Note that on $A_{1}(n+1) \supseteq A_{1}(\bar{n})$ we have $\varrho_{b}>\zeta_{n+1}$, for $b \in D_{n+1}$. Therefore, if $W_{s} \in D_{n+1}$ for $T_{n} \leq s \leq T_{n}+2^{-n}$, then $T_{n+1}=T_{n}+2^{-n}$ and

$$
\int_{T_{n}}^{T_{n+1}} L_{[W, \varrho]}(d s) \geq 2^{-n} \zeta_{n+1}=\xi_{n}
$$

[recall (182)]. Hence,

$$
\Pi_{T_{n}, a}\left(E_{n}^{\mathrm{c}}\right) \leq \Pi_{T_{n}, a}\left(W_{s} \notin D_{n+1} \text { for some } s \in\left[T_{n}, T_{n}+2^{-n}\right]\right), \quad a \in D_{n} .
$$

The strong Markov property applied to $T_{n}$ gives

$$
\Pi_{T_{n}, a}\left(E_{n}^{\mathrm{c}}\right) \leq \Pi_{0,0}\left(\sup _{s \leq 2^{-n}}\left|W_{s}\right|>2^{n}\right) .
$$

From our "traveling estimate" (169), it follows that for $N$ large enough, $n \geq N$ implies

$$
\Pi_{T_{n}, a}\left(E_{n}^{\mathrm{c}}\right) \leq\left(\frac{2^{-n} e}{2^{n}}\right)^{2^{n}}\left(2 \pi 2^{n}\right)^{-1 / 2} \leq c 2^{-2 \cdot 2^{n}} \leq \lambda_{n} \frac{M_{n+1}}{M_{n}} .
$$

Thus, Lemma 11 gives the conditional expectation estimate (44). 
Again, for $0 \leq s \leq 2^{-n}$, Proposition 12 yields

$$
\widetilde{P}_{0, \delta_{0}}^{\varrho}\left\{\tilde{X}_{T_{n}}^{\varrho}\left(E_{n}\right)>0 \mid\left\|\widetilde{X}_{T_{n}^{\varepsilon}}^{\varrho}\right\| \leq M_{n}\right\} \leq \frac{M_{n}}{\xi_{n}}=\delta_{n} .
$$

This proves the good paths estimate (43).

So Hypothesis 9 is satisfied, and finite time extinction for the lattice model follows from the abstract Theorem 10.

Acknowledgment. We thank an anonymous referee for carefully reading the manuscript and requesting the inclusion of some nontrivial counterexamples.

\section{REFERENCES}

[1] Barlow, M. T., Evans, S. N. and Perkins, E. A. (1991). Collision local times and measurevalued processes. Canad. J. Math. 43 897-938.

[2] Billingsley, P. (1986). Probability and Measure, 2nd ed. Wiley, New York.

[3] Dawson, D. A. and Fleischmann, K. (1991). Critical branching in a highly fluctuating random medium. Probab. Theory Related Fields 90 241-274.

[4] Dawson, D. A. and Fleischmann, K. (1992). Diffusion and reaction caused by point catalysts. SIAM J. Appl. Math. 52 163-180.

[5] Dawson, D. A. and Fleischmann, K. (1997). A continuous super-Brownian motion in a super-Brownian medium. J. Theoret. Probab. 10 213-276.

[6] Dawson, D. A. and Fleischmann, K. (1999). Catalytic and mutually catalytic branching. Preprint 510. WIAS Berlin.

[7] Dawson, D. A., Fleischmann, K. and Leduc, G. (1998). Continuous dependence of a class of superprocesses on branching parameters and applications. Ann. Probab. 26 262-601.

[8] Dawson, D. A., Fleischmann, K. and Roelly, S. (1991). Absolute continuity for the measure states in a branching model with catalysts. Prog. Probab. 24 117-160.

[9] Dawson, D. A., LI, Y. and Mueller, C. (1995). The support of measure-valued branching processes in a random environment. Ann. Probab. 23 1692-1718.

[10] Dawson, D. A. and Perkins, E. A. (1991). Historical processes. Mem. Amer. Math. Soc. 93 iv +179 .

[11] Donsker, M. D. and Varadhan, S. R. S. (1975). Asymptotic evaluation of certain Wiener integrals for large time. In Functional Integration and its Applications. Proceedings of the International Congress (A. M. Arthurs, ed.) 15-33. Clarendon Press, Oxford.

[12] Donsker, M. D. and Varadhan, S. R. (1977). On laws of the iterated logarithm for local times. Comm. Pure Appl. Math. 30 707-753.

[13] Dym, H. and McKean, H. P. (1972). Fourier Series and Integrals. Academic Press, New York.

[14] Dynkin, E. B. (1991). Branching particle systems and superprocesses. Ann. Probab. 19 $1157-1194$.

[15] Dynkin, E. B. (1991). Path processes and historical superprocesses. Probab. Theory Related Fields 90 1-36.

[16] Engländer, J. and Fleischmann, K. (2000). Extinction properties of super-Brownian motions with additional spatially dependent mass production. Stoch. Process. Appl. 88 37-58.

[17] Fleischmann, K. and Klenke, A. (1999). Smooth density field of catalytic super-Brownian motion. Ann. Appl. Probab. 9 298-318.

[18] Fleischmann, K. and Klenke, A. (2000). The biodiversity of catalytic super-Brownian motion. Ann. Appl. Probab. To appear.

[19] Fleischmann, K. and Le Gall, J.-F. (1995). A new approach to the single point catalytic super-Brownian motion. Probab. Theory Related Fields 102 63-82. 
[20] Fleischmann, K. and MuelleR, C. (1997). A super-Brownian motion with a locally infinite catalytic mass. Probab. Theory Related Fields 107 325-357.

[21] Fleischmann, K. and Mueller, C. (2000). Finite time extinction of catalytic branching processes. In Stochastic Models. A Conference in Honour of Donald A. Dawson (L. G. Gorostiza and B. G. Ivanoff, eds.) 125-139. Amer. Math. Soc., Providence, RI.

[22] FRISTEDT, B. E. (1967). Sample function behavior of increasing processes with stationary, independent increments. Pacific J. Math. 21 21-33.

[23] Galambos, J. (1978). The Asymptotic Theory of Extreme Order Statistics. Wiley, New York.

[24] GLITZKY, A., GRÖGER, K. and HÜNLICH, R. (1996). Free energy and dissipation rate for reaction diffusion processes of electrically charged species. Appl. Anal. 60 201-217.

[25] Klenke, A. (2000). A review on spatial catalytic branching. In Stochastic Models. A Conference in Honour of Donald A. Dawson (L. G. Gorostiza and B. G. Ivanoff, eds.) 245-263. Amer. Math. Soc., Providence, RI.

[26] Konno, N. and SHiga, T. (1988). Stochastic partial differential equations for some measurevalued diffusions. Probab. Theory Related Fields 79 201-225.

[27] ORtoleva, P. and Ross, J. (1972). Local structures in chemical reactions with heterogeneous catalysis. J. Chemical Phys. 56 4397-4400.

[28] Pagliaro, L. and TAYlor, D. L. (1988). Aldolase exists in both the fluid and solid phases of cytoplasm. J. Cell Biology 107 981-999.

[29] Perkins, E. A. (1995). On the martingale problem for interactive measure-valued branching diffusions. Mem. Amer. Math. Soc. 549.

[30] Revuz, D. and Yon, M. (1991). Continuous Martingales and Brownian Motion. Springer, Berlin.

[31] Shiga, T. and Shimizu, A. (1980). Infinite-dimensional stochastic differential equations and their applications. J. Math. Kyoto Univ. 20 395-416.

D. A. Dawson

FielDS InSTITUTE

222 College Street

TORONTO, ONTARIO

CANADA M5T 3J1

E-MAIL: ddawson@fields.utoronto.ca

\author{
K. FLEISCHMANN \\ WEIERSTRASS INSTITUTE \\ FOR APPLIED ANALYSIS AND STOCHASTICS \\ MOHRENSTR. 39 \\ D-10117 BERLIN \\ GERMANY \\ E-MAIL: fleischmann@wias_berlin.de
}

C. MUeller

DEPARTMENT OF MATHEMATICS

UNIVERSITY OF ROCHESTER

ROCHESTER, NEW YORK 14627

E-MAIL: cmlr@troi.cc.rochester.edu 